\title{
Time-delayed feedback control of dynamical small-world networks at Hopf bifurcation
}

\author{
Min Xiao • Daniel W.C. Ho · Jinde Cao
}

Received: 14 May 2008 / Accepted: 23 February 2009 / Published online: 17 March 2009

(C) The Author(s) 2009. This article is published with open access at Springerlink.com

\begin{abstract}
We show that time-delayed feedback methods, which have successfully been used to control unstable steady states or periodic orbits, provide a tool to control Hopf bifurcation for a small-world network model with nonlinear interactions and time delays. We choose the interaction strength parameter as a bifurcation parameter. Without control, bifurcation will occur
\end{abstract}

The research of M. Xiao was jointly supported by the project of Jiangsu Province of China, and the Fundamental Discipline Construction Foundation of Nanjing Xiaozhuang University. The research of D.W.C. Ho was supported by CityU SRG 7002355.

The research of J. Cao was jointly supported by the National Natural Science Foundation of China under Grant No. 60874088, the Specialized Research Fund for the Doctoral Program of Higher Education under Grant 20070286003, and 333 project of Jiangsu Province of China.

M. Xiao $(\bowtie) \cdot$ J. Cao

Department of Mathematics, Southeast University, Nanjing 210096, China

e-mail: candymanxm2003@yahoo.com.cn

J. Cao

e-mail: jdcao@seu.edu.cn

D.W.C. Ho

Department of Mathematics, City University

of Hong Kong, Hong Kong, China

e-mail:madaniel@cityu.edu.hk

M. Xiao

Department of Mathematics, Nanjing Xiaozhuang

University, Nanjing 210017, China early; meanwhile, the model can maintain a stationary total influenced volume only in a certain domain of the interaction strength parameter. However, outside of this domain the model still possesses a stable total influenced volume that can be guaranteed by delayed feedback perturbation, and the onset of the Hopf bifurcation is postponed. The feedback perturbation vanishes if the stabilization is successful and thus the domain of stability can be extended under only small control force. We present an analytical investigation of the feedback scheme using characteristic equation and discuss effects of both a low-pass filter included in the control loop and nonzero latency times associated with generation and injection of the feedback signal.

Keywords Small-world network · Hopf bifurcation . Time-delayed feedback - Bifurcation control ·

Latency time $\cdot$ Low-pass filter

\section{Introduction}

We have witnessed rapidly growing interest in control of nonlinear dynamic systems exhibiting bifurcation phenomena in recent years. There has been a wide variety of promising application of bifurcation controls. These include stall of compression system in jet engines, high incidence flight, voltage collapse in power systems, oscillatory behavior of tethered satellites, magnetic bearing systems, rotating chains, thermal convection loop, and cardiac alternates and heart 
rhythms, see $[1,2]$ and references therein. The aim of bifurcation control is to design a controller to modify the bifurcation properties of a given nonlinear system, thereby to achieve some desirable dynamical behaviors, see [1-7]. Typical objectives of bifurcation control are often achieved by delaying the onset of an inherent bifurcation, stabilizing an unstable bifurcated solution or branch, and changing the critical values of an existing bifurcation, etc.; see [2].

Various methods have been used to control and anti-control bifurcations in both discrete and continuous systems. Bifurcation control has been designed for stationary [31, 32], Hopf bifurcations [3, 33], perioddoubling bifurcations [34] and chaotic motions [8], etc. For Hopf bifurcation control, a static state feedback controller was proposed by Abed and $\mathrm{Fu}$ [32]. Later, nonlinear feedback (mainly cubic) controller for Hopf bifurcation was considered by Yagnoobi and Abed [35]. The main disadvantage of those controllers is that the controllers not only affect the stability of the equilibrium being controlled, but also may change the location and stability of other equilibria. Thus, control energy is wasted in the forced alteration of the system equilibrium, and moreover the system performance is often degraded due to being operated at an equilibrium different from the one designed to operate. Recently, Chen et al. [2] developed a dynamic state feedback control law incorporating a washout filter to control Hopf bifurcation in the Lorenz system. Later the washout filter controller was applied by Wen et al. to control and anti-control Hopf bifurcation in discrete maps [36]. The advantage of the washout filter-aided controller is to preserve all the systems equilibria, but it increases the dimension of the system by one, which not only changes the structure of the original system, but also increases the complexity of dynamical analysis. Moreover, the washout filter controller may destroy the symmetry of the equilibria of the original system [5]. Time-delayed feedback control has also been widely used in controlling chaos. Bleich and Socolar [8] used time-delayed feedback to obtain stable periodic orbits in a chaotic system, while Brandt et al., in [9], designed a linear, time-delayed feedback control for suppressing a pathological period-2 rhythm in an atrioventricular nodal conduction model. Recently, Song et al., in [10], proposed a chaos control method using time-delay based on repetitive learning. However, it has been noted that the application of timedelayed feedback in controlling bifurcations is not so popular, especially for controlling bifurcations arising from time-delayed systems. In this paper, timedelayed feedback will be extended to consider control of Hopf bifurcations in a delayed systems.

Starting with the work of Watts and Strogatz [11] on small-world networks, a lot of interesting researches on the theory and application of small-world networks [12-16] have arisen. The properties of complicated networks, such as internet servers, power grids, forest fires, and disordered porous media, are mainly determined by the way of connections between the vertices or occupied sites. One limiting case is the regular network with a high degree of local clustering and a large average distance, while the other limiting case is the random network with negligible local clustering and a small average distance. The small-world network is a special class of networks with a high degree of local clustering as well as a small average distance. This a small-world phenomenon can be found in many common networks such as the Internet, power grids, financial networks, and neural networks.

Recently, Newman and Watts [12] and Moukarzel [13] studied the spreading and response of an influence in a system with sparse long-range connections by using the small-world model. The Newman-Watts and Moukarzel models are linear models in the sense that the governing equation is linear and the response is immediate as there is no time delay in the models. However, in reality, there are usually time delays in spreading and response. Also, the nonlinear interactions due to competition and communication congestion of vertices should be modelled in order to simulate more realistic networks. Thus, in [17], a more general nonlinear delayed differential equation model was formulated for small-world networks, as

$\frac{\mathrm{d}^{d} V}{\mathrm{~d} t^{d}}=\xi^{d}+V(t-\tau)-\mu \xi^{d} V^{2}(t-\tau)$,

where $V(t)$ is the total influenced volume, $d$ is the dimension of the network, $\xi$ is the Newman-Watts length scale [12], and $\mu$ is a measure of nonlinear interactions in the network. By recasting (1) into a nonlinear difference equation, Yang [17] investigated the chaotic behavior as well as its control of this network model in the one-dimensional case $(d=1)$. Li et al. [18], on the other hand, showed that Hopf bifurcation may occur as the measure parameter, $\mu$, passes through a critical point, where a family of periodic solutions bifurcate from an equilibrium point. 
In this paper, bifurcation control using a timedelayed feedback controller for the delayed differential equation (1), also in one dimension case $(d=1)$, will be considered. This system can be described as

$$
\frac{\mathrm{d} V}{\mathrm{~d} t}=\xi+V(t-\tau)-\mu \xi V^{2}(t-\tau) .
$$

The study of bifurcation control in a small-world network model is quite significant. On the one hand, the bifurcations, which involve emergence of oscillatory behaviors, may provide an explanation for the parameter sensitivity observed in practice in many realistic small-world networks such as the Internet, the electrical power grids, and the biological neural networks; and on the other hand, based on the bifurcation of small-world networks, we can apply the existing effective bifurcation control methods [19] to achieve some desirable system behaviors that benefit the networks. In detail, Hopf bifurcation may occur as $\mu$ passes through a critical point. Thus, the total influenced volume $V$ is not guaranteed, which is not desirable. In this paper, our objective here is to design a delayed feedback controller to introduce a new Hopf bifurcation with regard to $\mu$ as well as make the Hopf bifurcation to have certain characteristics. We will delay the onset of Hopf bifurcation by adding time-delayed feedback control to the model (2). We will show, with a Hopf bifurcation controller, that one can increase the critical value of measure parameter, $\mu$, thereby, guarantee a stationary total influenced volume for large parameter values, which benefits small-world networks.

The main contribution of this paper is design a general time-delayed feedback scheme to control Hopf bifurcation for the small-world network model, which is valid for any dynamical system close to the bifurcation point. This delayed feedback controller keeps the equilibria of a system unchanged and also preserves the dimension of the system. Thus, the controlled system has the same structure as the original system. Although some methods can achieve the bifurcation control in most researches, controller designs appear purely arbitrary and complex, and the characteristic of original system is destroyed. Therefore, bifurcation control can be only realized in mathematical viewpoint. There is no link between the controllers and the physical configuration. From the control theory viewpoint, the realistic problem of bifurcation control is to design a feedback scheme such that less possible simple of controller be interconnected. Our controller is based on feedback control strategy, thus, bifurcation control can be carried out by physical configuration. The results obtained are of general importance for optimizing the control technique of Hopf bifurcation and stimulate the search for further modifications aiming at the improvement of the control performance.

The organization of this article is as follows. In the next section, the main results for the Hopf bifurcation of the small-world network model obtained in [18] are summarized for completeness and convenience. In Sect. 3, we will introduce the control force and investigate the domain of stability, the onset and direction of bifurcation using characteristic equation. In Sect. 4, we will discuss the extended delayed feedback. Further, we will consider the effects of nonzero latency times and additional low-pass filtering in Sects. 5 and 6. Finally, a conclusion is drown in Sect. 7.

\section{Stability and bifurcation of uncontrolled system}

In this section, the results of stability and Hopf bifurcation in the small-world network model, obtained in [18], are summarized here for comparison, completeness and convenience.

Theorem 1 For system (2), the positive equilibrium $V^{*}$ is locally stable if $\mu<\frac{\pi^{2}-4 \tau^{2}}{16 \tau^{2} \xi^{2}}$, and unstable if $\mu>$ $\frac{\pi^{2}-4 \tau^{2}}{16 \tau^{2} \xi^{2}}$, where $V^{*}=\frac{1+\sqrt{1+4 \mu \xi^{2}}}{2 \mu \xi}$ (see [18]).

From the conclusion of Theorem 1, the bifurcation diagram is drawn in the parameter plane $(\tau, \mu)$ as in Fig. 1.

Theorem 2 For system (2), a Hopf bifurcation occurs from its positive equilibrium, $V^{*}$, when the measure parameter, $\mu$, passes through the critical value, $\mu^{*}=$ $\frac{\pi^{2}-4 \tau^{2}}{16 \tau^{2} \xi^{2}}$, where $V^{*}=\frac{1+\sqrt{1+4 \mu \xi^{2}}}{2 \mu \xi}($ see $[18])$.

Theorem 3 The Hopf bifurcation in the small-world network model (2) is determined by the parameters $\mu_{2}, \beta_{2}, \tau_{2}$, where $\mu_{2}$ determines the direction of the Hopf bifurcation: if $\mu_{2}>0\left(\mu_{2}<0\right)$, then the Hopf bifurcation is supercritical (subcritical) and the bifurcating periodic solutions exist for $\mu>\mu^{*}\left(\mu<\mu^{*}\right)$; $\beta_{2}$ determines the stability of the bifurcating periodic solutions: the bifurcating periodic solutions are stable (unstable) if $\beta_{2}<0\left(\beta_{2}>0\right)$; and $\tau_{2}$ determines the 
Fig. 1 The curve $\mu=\frac{\pi^{2}-4 \tau^{2}}{16 \tau^{2} \xi^{2}}$ divides the first quadrant of $(\tau, \mu)$-plane into two regions, $D_{1}$ and $D_{2} . D_{2}$ is an absolutely stable region; $D_{1}$ is an unstable region

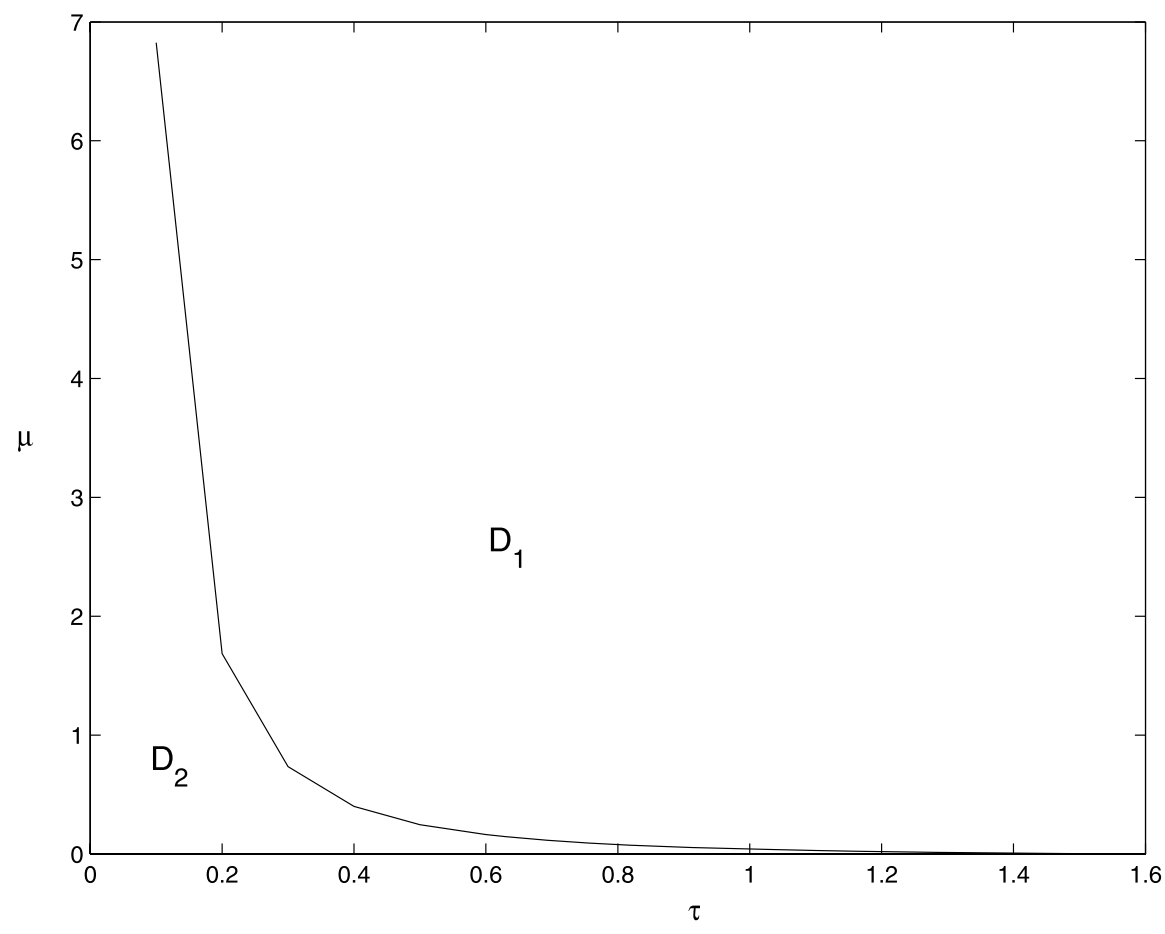

period of the bifurcating periodic solutions: the period increases (decreases) if $\tau_{2}>0\left(\tau_{2}<0\right)$.

The parameters $\mu_{2}, \beta_{2}, \tau_{2}$ are given by

$\mu_{2}=-\frac{\operatorname{Re}\left\{C_{1}(0)\right\}}{\operatorname{Re}\left\{\lambda^{\prime}(0)\right\}}$,

$\beta_{2}=2 \operatorname{Re}\left\{C_{1}(0)\right\}$,

$\tau_{2}=-\frac{\operatorname{Im}\left\{C_{1}(0)\right\}+\mu_{2} \operatorname{Im}\left\{\lambda^{\prime}(0)\right\}}{\omega_{0}}$,

where

$\operatorname{Re}\left\{\lambda^{\prime}(0)\right\}=\frac{2 \xi^{2} \tau}{1+\left(b_{1}^{*} \tau\right)^{2}}$,

$\operatorname{Im}\left\{\lambda^{\prime}(0)\right\}=-\frac{1}{b_{1}^{*} \tau} \frac{\mathrm{d} \alpha}{\mathrm{d} \mu}=-\frac{2 \xi^{2}}{\left[1+\left(b_{1}^{*} \tau\right)^{2}\right] b_{1}^{*}}$,

$C_{1}(0)=\frac{\mathrm{i}}{2 \omega_{0}}\left(g_{11} g_{20}-2\left|g_{11}\right|^{2}-\frac{\left|g_{02}\right|^{2}}{3}\right)+\frac{g_{21}}{2}$,

in which

$$
\begin{aligned}
\omega_{0} & =\frac{\pi}{2 \tau}, \\
g_{20} & =g_{02}=-g_{11}=\frac{-2 b_{2}^{*}}{1+b_{1}^{*} \tau e^{-\mathrm{i} \omega_{0} \tau}},
\end{aligned}
$$

$$
\begin{aligned}
g_{21}= & \frac{2 \mathrm{i}}{1+b_{1}^{*} \tau e^{-\mathrm{i} \omega_{0} \tau}}\left[\frac{2\left(2 b_{2}^{*}-g_{11}-\bar{g}_{11}\right) b_{2}^{*}}{b_{1}^{*}}\right. \\
& \left.+\frac{\left(g_{20}-\bar{g}_{02}+2 b_{2}^{*}\right) b_{2}^{*}}{b_{1}^{*}-2 \mathrm{i} \omega_{0}}\right], \\
b_{1}^{*}= & -\sqrt{1+4 \mu^{*} \xi^{2}}, \\
b_{2}^{*}= & -\mu^{*} \xi .
\end{aligned}
$$

The detailed derivation of the above formulas can be found in [18].

\section{Control by time-delayed feedback}

We now turn to design a time-delayed feedback force to accomplish the control of the Hopf bifurcation arising from the small-world network model (2). Following the ideal of Pyragas [20], we add a time-delayed controller $\alpha(V(t-\tau)-V(t))$ to the model (2), that is the following delayed feedback control system:

$$
\begin{aligned}
\frac{\mathrm{d} V}{\mathrm{~d} t}= & \xi+V(t-\tau)-\mu \xi V^{2}(t-\tau) \\
& +\alpha(V(t-\tau)-V(t))
\end{aligned}
$$


where the feedback gain $\alpha$ is real number. It is easy to see that this time-delayed feedback controller preserves the equilibrium point of the small-world network model (2). The goal of the control is to change the Hopf bifurcation to achieve desirable behaviors, such as delaying the onset of a Hopf bifurcation.

Remark 1 In [21], Cheng and Cao proposed the following delayed feedback:

$$
\begin{aligned}
& \alpha_{1}\left(V(t-\tau)-V^{*}\right)+\alpha_{2}\left(V(t-\tau)-V^{*}\right)^{2} \\
& \quad+\alpha_{3}\left(V(t-\tau)-V^{*}\right)^{3},
\end{aligned}
$$

to control the Hopf bifurcation of the small-world network model (2). However, for some systems, the natural equilibrium cannot be given by analytic expression. Therefore, delayed feedback controller in [21] are unable to be added to this kind of system. In this paper, a new time-delayed feedback controller will be extended to consider control of Hopf bifurcations in the small-world network model. This controller is more general in bifurcation control and valid for any dynamical system close to the bifurcation point.

\subsection{Stability and existence of bifurcation}

Using Taylor expansion, we can expand the right-hand side of (6) around $V^{*}$, resulting in the following linearized equation:

$\dot{V}(t)=-\alpha\left(V(t)-V^{*}\right)+b_{1}\left(V(t-\tau)-V^{*}\right)$,

where $b_{1}=\alpha-\sqrt{1+4 \mu \xi^{2}}$. Let $u(t)=V(t)-V^{*}$, then (7) becomes

$\dot{u}(t)=-\alpha u(t)+b_{1} u(t-\tau)$,

which has the characteristic equation:

$\lambda+\alpha-b_{1} e^{-\lambda \tau}=0$.

In what follows, we regard $\mu$ as the bifurcation parameter to investigate the distribution of the roots to (9).

Lemma 4 If $\alpha>0$, we have

(i) When $0<\mu \leq \frac{4 \alpha^{2}-1}{4 \xi^{2}}$, all the roots of (9) have negative real parts. (ii) When $\mu>\frac{4 \alpha^{2}-1}{4 \xi^{2}}$, there exists $\tau_{0}$ such that all the roots of (9) have negative real parts when $\tau \in\left[0, \tau_{0}\right)$, and (9) has at least one root with positive real part when $\tau>\tau_{0}$, where

$$
\tau_{0}=\frac{1}{\sqrt{b_{1}^{2}-\alpha^{2}}} \arccos \left(\frac{\alpha}{b_{1}}\right) .
$$

Proof (i) When $b_{1}=0, \lambda=-\alpha<0$, let $\mathrm{i} \omega$ (i is the unit of imaginary part, $\omega>0$ ) be a root of (9), it is straightforward to obtain that

$b_{1} \cos (\omega \tau)-\alpha=0, \quad \omega+b_{1} \sin (\omega \tau)=0$,

yielding $\omega^{2}=b_{1}^{2}-\alpha^{2}$, which dose not hold when $\left|b_{1}\right|<\alpha$. When $b_{1}=-\alpha$, for (8), there is no pure imaginary root, and $\lambda=0$ is not its root. These imply that there is no root appearing on the imaginary axis when $b_{1} \in[-\alpha, \alpha)$. Thus, all the roots of (9) have negative real parts when $b_{1} \in[-\alpha, \alpha)$.

Since $0<\mu \leq \frac{4 \alpha^{2}-1}{4 \xi^{2}}$ is equivalent to $b_{1} \in[-\alpha, \alpha)$, the conclusion (i) follows.

(ii) If $\mu>\frac{4 \alpha^{2}-1}{4 \xi^{2}}$ holds, we have $b_{1}<-\alpha$. From the definition of $\tau_{0}$, we know that $\left(\tau_{0}, \sqrt{b_{1}^{2}-\alpha^{2}}\right)$ is a solution of (9), i.e., $\pm \mathrm{i} \sqrt{b_{1}^{2}-\alpha^{2}}$ is a pair of pure imaginary roots of (9) with $\tau=\tau_{0}$.

Clearly, $\tau_{0}$ is the first value of $\tau>0$ such that (9) has root appearing on the imaginary axis. Therefore, all the roots of (9) have negative real parts when $\tau \in$ $\left[0, \tau_{0}\right)$.

Let $\lambda(\tau)=\rho(\tau)+\mathrm{i} \omega(\tau)$ be the root of (9) satisfying $\rho\left(\tau_{0}\right)=0$ and $\omega\left(\tau_{0}\right)=\sqrt{b_{1}^{2}-\alpha^{2}}$. We can obtain

$\frac{\mathrm{d} \lambda}{\mathrm{d} \tau}=-\frac{b_{1} \lambda e^{-\lambda \tau}}{1+b_{1} \tau e^{-\lambda \tau}}$,

then

$\rho^{\prime}\left(\tau_{0}\right)=\operatorname{Re}\left(\left.\frac{\mathrm{d} \lambda}{\mathrm{d} \tau}\right|_{\tau=\tau_{0}}\right)=\frac{\omega^{2}}{\left(1-\alpha \tau_{0}\right)^{2}+\left(\omega \tau_{0}\right)^{2}}>0$.

Thus, (9) has at least one root with positive real part when $\tau>\tau_{0}$. The conclusion (ii) follows. Then the proof is completed.

From Lemma 4, we easily obtain the following results about the stability of the equilibrium $V^{*}$ of system (6). 
Fig. 2 The curve

$\mu=\frac{4 \alpha^{2}-1}{4 \xi^{2}}$ divides the first quadrant of $(\alpha, \mu)$-plane into two regions, $S_{1}$ and $S_{2}$. $S_{2}$ is an absolutely stable region; $S_{1}$ is a conditionally stable region, and there is a stability switch for the parameter being located in the region $S_{1}$

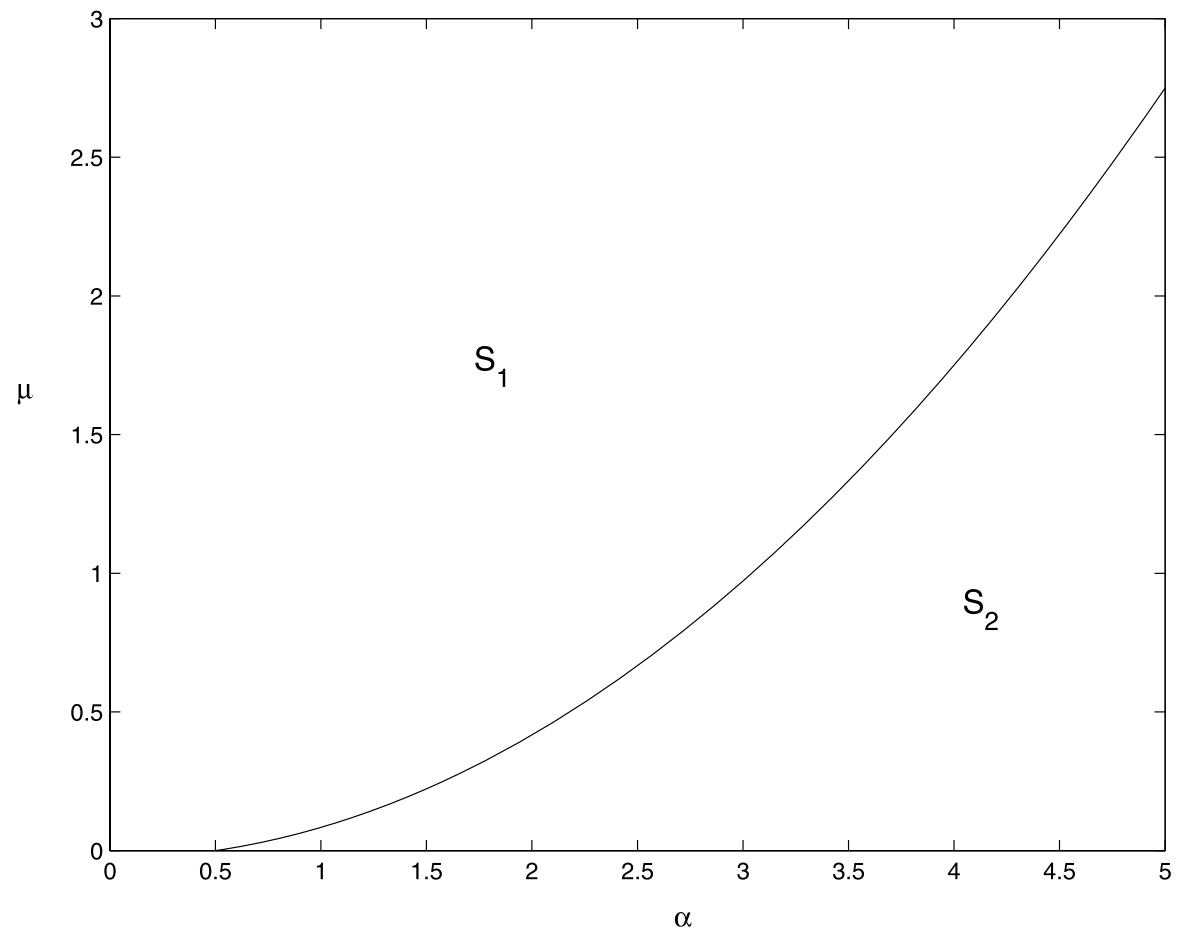

Theorem 5 Let $\alpha>0$, we have

(i) If $0<\mu \leq \frac{4 \alpha^{2}-1}{4 \xi^{2}}$, then the equilibrium $V^{*}$ of system (6) is asymptotically stable for all $\tau \geq 0$.

(ii) If $\mu>\frac{4 \alpha^{2}-1}{4 \xi^{2}}$, then the $V^{*}$ is asymptotically stable when $\tau \in\left[0, \tau_{0}\right)$ and unstable when $\tau>\tau_{0}$.

Proof It is well known that the solution is locally asymptotically stable if all the roots of the characteristic equation have negative real parts and unstable if at least one root has positive real part. Therefore, conclusions (i) and (ii) are straightforward from Lemma 4. This completes the proof.

According to the conclusion of Theorem 5, we can draw the bifurcation diagram in the parameter plane $(\alpha, \mu)$ as in Fig. 2.

Lemma 6 For the controlled system (6), there exists a minimum positive number $\mu_{0}^{+}$such that (9) has a pair of purely imaginary roots $\pm \mathrm{i} \omega_{0}^{+}$at $\mu=\mu_{0}^{+}$.

Proof If $\lambda=\mathrm{i} \omega$ is a pure imaginary solution of (9), then it must satisfy (10). Then, we have the following equation:

$\tan (\omega \tau)=-\frac{\omega}{\alpha}$.

Let $v=\omega \tau$, then we have $\tan (v)=-v /(\alpha \tau)$. Solutions of this equation are the horizontal coordinates of the intersecting points between the curve $y=\tan (v)$ and the line $y=-v /(\alpha \tau)$. There are infinite number of intersecting points for these two curves that are graphically illustrated in Fig. 3.

Without loss of generality, we only consider the intersecting points with positive horizontal coordinates $v_{i}, i=1,2, \ldots$ It is clear that $\mathrm{i} \pi-\pi / 2<$ $v_{i}<\mathrm{i} \pi, i=1,2, \ldots$, and $\mathrm{i} \pi-v_{i} \rightarrow \pi / 2$ monotonically when $i \rightarrow \infty$. For these $v_{i}$, from (10), we can choose $b_{1}^{i}=\alpha / \cos \left(v_{i}\right)$, then we have $\left|b_{1}^{i}\right| \rightarrow+\infty$ and $b_{1}^{1}, b_{1}^{3}, b_{1}^{5}, \ldots<0, b_{1}^{2}, b_{1}^{4}, b_{1}^{6}, \ldots>0$. Then, from $b_{1}=\alpha-\sqrt{1+4 \mu \xi^{2}}$, we have

$\mu_{i}=\frac{\left(\alpha-b_{1}^{i}\right)^{2}-1}{4 \xi^{2}}$.

Since $\left|b_{1}^{i}\right|>\alpha$, we obtain the following orderings:

$0<\mu_{1}<\mu_{3}<\mu_{5}<\cdots, \quad$ and

$0<\mu_{2}<\mu_{4}<\mu_{6}<\cdots$. 
Fig. 3 Illustration for intersecting points between curve $y=\tan (v)$ and line $y=-v /(\alpha \tau)$

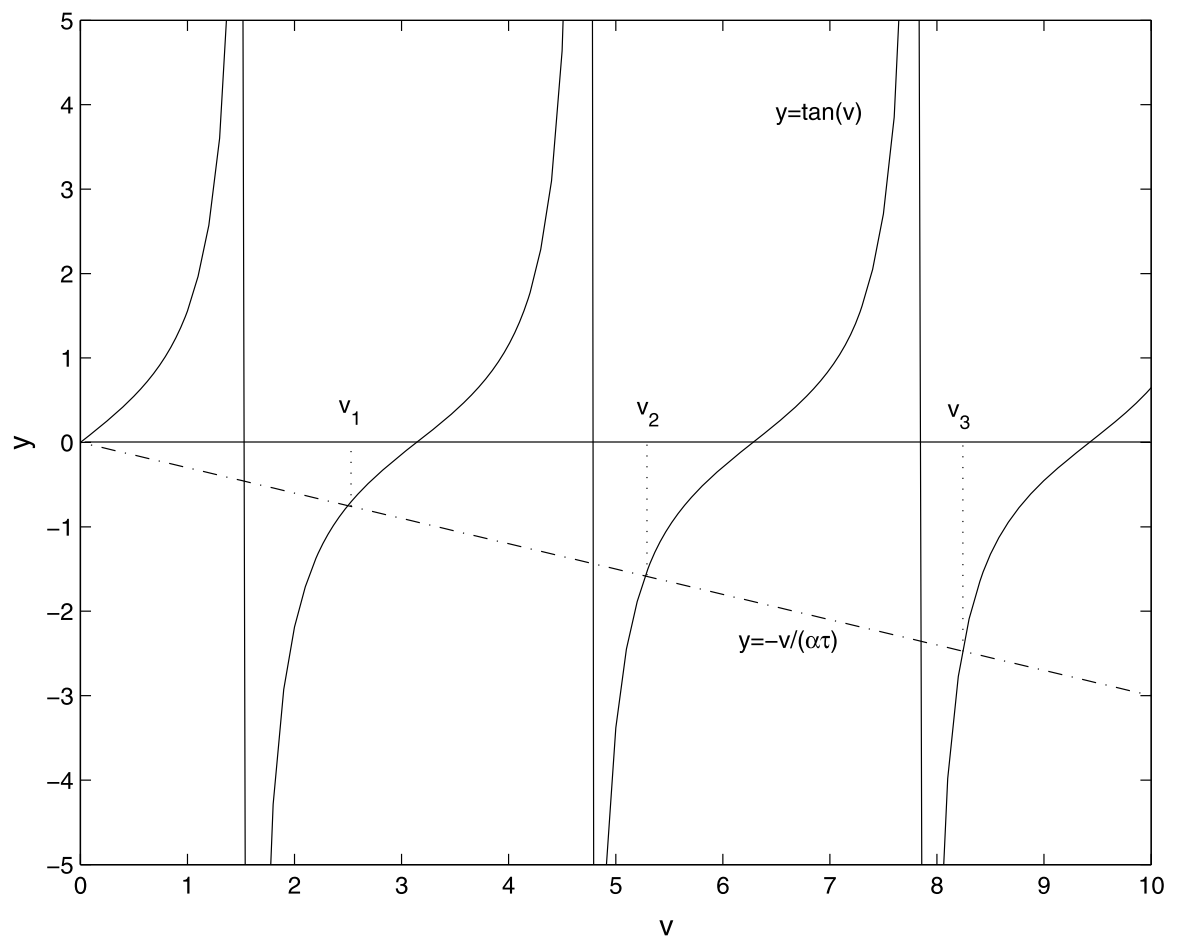

Thus, we can choose $\mu_{0}^{+}=\min _{i \in\{1,2\}}\left\{\mu_{i}\right\}=$ $\min _{i \in\{1,2\}}\left\{\frac{\left(\alpha-b_{1}^{i}\right)^{2}-1}{4 \xi^{2}}\right\}$. Clearly, (9) has a pair of purely imaginary roots $\pm \mathrm{i} \omega_{0}^{+}$at $\mu=\mu_{0}^{+}$.

Remark 2 It should be noted that in the proof of Lemma 6 , the two orderings of $\mu$ is indeed independent of $\alpha$.

We will only discuss the case of $\mu=\mu_{0}^{+}$. In this case, $\omega=\omega_{0}^{+}$. Then, we have the following results about Hopf bifurcation.

Theorem 7 For the controlled system (6), there exists a Hopf bifurcation emerging from its equilibrium $V^{*}$, when the measure parameter, $\mu$, passes through the critical value, $\mu^{*}=\mu_{0}^{+}$, where the equilibrium point $V^{*}$ is kept unchanged, where $V^{*}=\frac{1+\sqrt{1+4 \mu \xi^{2}}}{2 \mu \xi}$.

Proof The last condition for the occurrence of a Hopf bifurcation at $\mu_{0}^{+}$is

$\frac{\mathrm{d}}{\mathrm{d} \mu}[\operatorname{Re} \lambda]_{\mu_{0}^{+}} \neq 0$.
Letting $\lambda=\rho+\mathrm{i} \omega$, and then substituting $\lambda$ into the characteristic (9) yields

$$
(\rho+\mathrm{i} \omega)+\alpha-b_{1} e^{-\rho \tau}[\cos (\omega \tau)-\mathrm{i} \sin (\omega \tau)]=0
$$

from which one can easily obtain

$$
\begin{aligned}
& \frac{\mathrm{d} \rho}{\mathrm{d} \mu}+\frac{2 \xi^{2}}{\sqrt{1+4 \mu \xi^{2}}} e^{-\rho \tau} \cos (\omega \tau) \\
& \quad+\left(\alpha-\sqrt{1+4 \mu \xi^{2}}\right) \tau e^{-\rho \tau} \cos (\omega \tau) \frac{\mathrm{d} \rho}{\mathrm{d} \mu} \\
& \quad+\left(\alpha-\sqrt{1+4 \mu \xi^{2}}\right) \tau e^{-\rho \tau} \sin (\omega \tau) \frac{\mathrm{d} \omega}{\mathrm{d} \mu}=0,
\end{aligned}
$$

$$
\begin{aligned}
& \frac{\mathrm{d} \omega}{\mathrm{d} \mu}-\frac{2 \xi^{2}}{\sqrt{1+4 \mu \xi^{2}}} e^{-\rho \tau} \sin (\omega \tau) \\
& -\left(\alpha-\sqrt{1+4 \mu \xi^{2}}\right) \tau e^{-\rho \tau} \sin (\omega \tau) \frac{\mathrm{d} \rho}{\mathrm{d} \mu} \\
& +\left(\alpha-\sqrt{1+4 \mu \xi^{2}}\right) \tau e^{-\rho \tau} \cos (\omega \tau) \frac{\mathrm{d} \omega}{\mathrm{d} \mu}=0 .
\end{aligned}
$$


This implies that

$\left.\frac{\mathrm{d} \rho}{\mathrm{d} \mu}\right|_{\mu=\mu_{0}^{+}}= \begin{cases}\frac{-\frac{2 \xi^{2}}{\sqrt{1+4 \mu_{0}^{+} \xi^{2}}}\left[\cos \left(v_{1}\right)+b_{1}^{1} \tau\right]}{\left[1+b_{1}^{1} \tau \cos \left(v_{1}\right)\right]^{2}+\left[b_{1}^{1} \tau \sin \left(v_{1}\right)\right]^{2}}, & \mu_{0}^{+}=\mu_{1}, \\ \frac{-\frac{2 \xi^{2}}{\sqrt{1+4 \mu_{0}^{+} \xi^{2}}}\left[\cos \left(v_{2}\right)+b_{1}^{2} \tau\right]}{\left[1+b_{1}^{2} \tau \cos \left(v_{2}\right)\right]^{2}+\left[b_{1}^{2} \tau \sin \left(v_{2}\right)\right]^{2}}, & \mu_{0}^{+}=\mu_{2},\end{cases}$

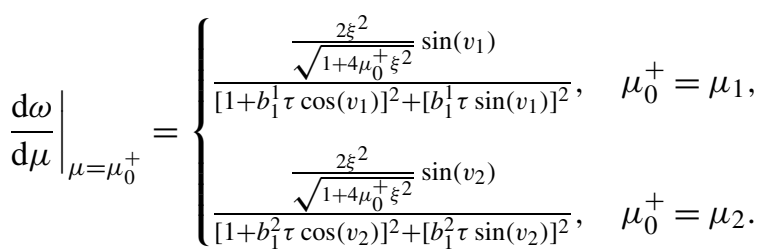

From the proof of Lemma 6 , since $\pi / 2<v_{1}<\pi$, we can obtain $\cos \left(v_{1}\right)<0, b_{1}^{1}=\alpha / \cos \left(v_{1}\right)<0$. Since $3 \pi / 2<v_{2}<2 \pi$, we can also obtain $\cos \left(v_{2}\right)>0$, $b_{1}^{2}=\alpha / \cos \left(v_{1}\right)>0$. Therefore, we have

$\left.\frac{\mathrm{d} \rho}{\mathrm{d} \mu}\right|_{\mu=\mu_{0}^{+}} \begin{cases}>0, & \mu_{0}^{+}=\mu_{1}, \\ <0, & \mu_{0}^{+}=\mu_{2} .\end{cases}$

Thus, the transversality condition (13) for Hopf bifurcation is satisfied. Applying Lemma 6 and Hopf bifurcation theorems for functional differential equations in [22], we obtain that Hopf bifurcation occurs at $\mu^{*}=\mu_{0}^{+}$for the controlled system (6). This completes the proof.

Remark 3 Theorem 7 shows that one can delay or advance the onset of a Hopf bifurcation without changing the original equilibrium points by choosing an appropriate value of $\alpha$.

\subsection{Stability of bifurcating periodic solutions}

Next, we will use the center manifold and normal form theories introduced in [23] to study the direction of Hopf bifurcation and stability of the bifurcating periodic solutions on the center manifold in the controlled system (6).

For notation convenience, let $\mu=\mu^{*}+\epsilon$. Then, $\epsilon=0$ is the Hopf bifurcation value for system (6). Applying Taylor expansion to the right-hand side of system (6) at the equilibrium point, $V^{*}$, we have

$\dot{u}(t)=-\alpha u(t)+b_{1} u(t-\tau)+b_{2} u^{2}(t-\tau)+o\left(|u|^{3}\right)$, where

$$
\begin{aligned}
& b_{1}=\alpha-\sqrt{1+4 \mu \xi^{2}}, \\
& b_{2}=-\mu \xi .
\end{aligned}
$$

For initial condition $\phi=\phi(s, \mu), s \in[-\tau, 0]$ with $\phi \in$ $C([-\tau, 0], \mathrm{R})$, we set

$L_{\mu} \phi=-\alpha \phi(0)+b_{1} \phi(-\tau)$,

and

$F(\mu, \phi)=b_{2} \phi^{2}(-\tau)+o\left(|\phi|^{3}\right)$.

By the Riesz representation theorem, there exists a function $\eta(\theta, \mu)$ of bounded variation for $\theta \in[-\tau, 0]$, such that

$L_{\mu} \phi=\int_{-\tau}^{0} \mathrm{~d} \eta(\theta, \mu) \phi(\theta) \quad$ for $\phi \in C$,

which can be satisfied by choosing

$\eta(\theta, \mu)=-\alpha \delta(\theta)+b_{1} \delta(\theta+\tau)$,

where $\delta$ is the Dirac delta function.

For $\phi \in C^{1}([-\tau, 0], \mathrm{R})$, define

$A(\mu) \phi= \begin{cases}\frac{\mathrm{d} \phi(\theta)}{\mathrm{d} \theta}, & \theta \in[-\tau, 0), \\ \int_{-\tau}^{0} \mathrm{~d} \eta(\mu, s) \phi(s), & \theta=0,\end{cases}$

and

$R(\mu) \phi= \begin{cases}0, & \theta \in[-\tau, 0), \\ F(\mu, \phi), & \theta=0 .\end{cases}$

Then, system (15) is equivalent to

$\dot{u}_{t}=A(\mu) u_{t}+R(\mu) u_{t}$,

where $u_{t}(\theta)=u(t+\theta)$ for $\theta \in[-\tau, 0]$.

For $\psi \in C([0, \tau], \mathrm{R})$, define

$A^{*} \psi(s)= \begin{cases}-\frac{\mathrm{d} \psi(s)}{\mathrm{d} s}, & s \in(0, \tau], \\ \int_{-\tau}^{0} \psi(-t) \mathrm{d} \eta(t, 0), & s=0,\end{cases}$

and a bilinear inner product 


$$
\begin{aligned}
& \langle\psi(s), \phi(\theta)\rangle \\
& =\bar{\psi}(0) \phi(0)-\int_{\theta=-\tau}^{0} \int_{\xi=0}^{\theta} \bar{\psi}(\xi-\theta) \mathrm{d} \eta(\theta) \phi(\xi) \mathrm{d} \xi,
\end{aligned}
$$

where $\eta(\theta)=\eta(\theta, 0)$. Then, $A(0)$ and $A^{*}$ are adjoint operators.

In order to determine the Poincare normal form of the operator $A(0)$, we need to calculate the eigenvector $q$ of $A(0)$ corresponding to the eigenvalue $\mathrm{i} \omega_{0}^{+}$and the eigenvector $q^{*}$ of $A^{*}$ corresponding to the eigenvalue $-\mathrm{i} \omega_{0}^{+}$. We can easily verify that

$q(\theta)=\exp \left(\mathrm{i} \omega_{0}^{+} \theta\right), \quad \theta \in[-\tau, 0)$,

is the eigenvector of $A(0)$ corresponding to the eigenvalue $\mathrm{i} \omega_{0}^{+}$, and

$q^{*}(s)=B \exp \left(\mathrm{i} \omega_{0}^{+} s\right), \quad s \in[0, \tau)$,

is the eigenvector of $A^{*}$ corresponding to $-\mathrm{i} \omega_{0}^{+}$.

From (18), we have

$$
\begin{aligned}
& \left\langle q^{*}(s), q(\theta)\right\rangle \\
& \quad=\bar{B}-\int_{\theta=-\tau}^{0} \int_{\xi=0}^{\theta} \bar{B} e^{-\mathrm{i} \omega_{0}^{+}(\xi-\theta)} \mathrm{d} \eta(\theta) e^{\mathrm{i} \omega_{0}^{+} \xi} \mathrm{d} \xi \\
& \quad=\bar{B}-\bar{B} \int_{\theta=-\tau}^{0} \theta e^{\mathrm{i} \omega_{0}^{+} \theta} \mathrm{d} \eta(\theta) \\
& \quad=\bar{B}\left(1+b_{1} \tau e^{-\mathrm{i} \omega_{0}^{+} \tau}\right) .
\end{aligned}
$$

Thus, we can choose

$$
B=\frac{1}{1+b_{1} \tau e^{\mathrm{i} \omega_{0}^{+} \tau}},
$$

such that $\left\langle q^{*}(s), q(\theta)\right\rangle=1$.

Following the algorithms given in [23] and using a computation process similar to that in [24], we can obtain the coefficient which will be used in determining the important quantities:

$$
\begin{aligned}
& g_{20}=-2 \bar{B} \mu \xi e^{-2 \mathrm{i} \omega_{0}^{+} \tau}, \\
& g_{11}=-2 \bar{B} \mu \xi \\
& g_{02}=-2 \bar{B} \mu \xi e^{2 \mathrm{i} \omega_{0}^{+} \tau}, \\
& g_{21}=-2 \bar{B} \mu \xi\left[2 e^{-\mathrm{i} \omega_{0}^{+} \tau} W_{11}(-\tau)+e^{\mathrm{i} \omega_{0}^{+} \tau} W_{20}(-\tau)\right],
\end{aligned}
$$

where

$$
\begin{aligned}
& W_{20}(\theta)=\frac{\mathrm{i} g_{20}}{\omega_{0}^{+}} e^{\mathrm{i} \omega_{0}^{+} \theta}+\frac{\mathrm{i} \bar{g}_{02}}{3 \omega_{0}^{+}} e^{-\mathrm{i} \omega_{0}^{+} \theta}+E_{1} e^{2 \mathrm{i} \omega_{0}^{+} \theta}, \\
& W_{11}(\theta)=-\frac{\mathrm{i} g_{11}}{\omega_{0}^{+}} e^{\mathrm{i} \omega_{0}^{+} \theta}+\frac{\mathrm{i} \bar{g}_{11}}{\omega_{0}^{+}} e^{-\mathrm{i} \omega_{0}^{+} \theta}+E_{2},
\end{aligned}
$$

in which

$$
\begin{aligned}
& E_{1}=-\frac{2 \mu \xi e^{-2 \mathrm{i} \omega_{0}^{+} \tau}}{2 \mathrm{i} \omega_{0}^{+}+\alpha-b_{1} e^{-2 \mathrm{i} \omega_{0}^{+} \tau}}, \\
& E_{2}=-\frac{2 \mu \xi}{\alpha-b_{1}} .
\end{aligned}
$$

Therefore, each $g_{i j}$ in (19) has been expressed in terms of the parameters and the delay given in system (6). Furthermore, we can compute the following quantities:

$$
C_{1}(0)=\frac{\mathrm{i}}{2 \omega_{0}^{+}}\left(g_{11} g_{20}-2\left|g_{11}\right|^{2}-\frac{\left|g_{02}\right|^{2}}{3}\right)+\frac{g_{21}}{2},
$$

$\mu_{2}=-\frac{\operatorname{Re}\left\{C_{1}(0)\right\}}{\operatorname{Re}\left\{\lambda^{\prime}(0)\right\}}$,

$\beta_{2}=2 \operatorname{Re}\left\{C_{1}(0)\right\}$,

$\tau_{2}=-\frac{\operatorname{Im}\left\{C_{1}(0)\right\}+\mu_{2} \operatorname{Im}\left\{\lambda^{\prime}(0)\right\}}{\omega_{0}^{+}}$.

Now, the main results of this section are summarized as follows.

Theorem 8 The Hopf bifurcation exhibited by the controlled Internet congestion model (6) is determined by the parameters $\mu_{2}, \beta_{2}, \tau_{2}$, where $\mu_{2}$ determines the direction of the Hopf bifurcation: if $\mu_{2}>0\left(\mu_{2}<\right.$ $0)$, then the Hopf bifurcation is supercritical (subcritical) and the bifurcating periodic solutions exist for $\mu>\mu^{*}\left(\mu<\mu^{*}\right) ; \beta_{2}$ determines the stability of the bifurcating periodic solutions: the bifurcating periodic solutions are stable (unstable) if $\beta_{2}<0\left(\beta_{2}>0\right)$; and $\tau_{2}$ determines the period of the bifurcating periodic solutions: the period increases (decreases) if $\tau_{2}>0\left(\tau_{2}<0\right)$.

Remark 4 It is shown in Theorem 8 that besides changing the onset of a Hopf bifurcation without destroying the structure of uncontrolled system (2), direction of Hopf bifurcation and stability of the bifurcating periodic solutions can also be modulated by varying the value of the feedback gain parameter $\alpha$. 


\subsection{A numerical example}

In this section, we present numerical results to verify the analytical predictions obtained in the previous section, using the time-delayed feedback controller to control the Hopf bifurcation of small-world network model (2). The numerical approach is based on a fourth-order Runge-Kutta integration scheme.

For a consistent comparison, the same model (2), used in [18], is discussed, with $\xi=3$ and $\tau=1$. For the uncontrolled model, it follows from Theorems 2 and 3 that

$\mu^{*}=0.0408, \quad \omega_{0}=1.5708$,

and

$\mu_{2}=0.0019, \quad \tau_{2}=0.0033, \quad \beta_{2}=-0.0280$.

The dynamical behavior of this uncontrolled smallworld network model (2) is illustrated in Figs. 4-6. From Theorem 1, it is shown that when $\mu<\mu^{*}$, trajectories converge to the equilibrium point (see Fig. 4), while as $\mu$ is increased to pass $\mu^{*}, V^{*}$ loses its stability and a Hopf bifurcation occurs (see Figs. 5 and 6). Note that the periodic orbits are stable since $\beta_{2}<0$, the bifurcating periodic solutions exist at least for the value $\mu$ slightly larger than the critical value $\mu^{*}$ since $\mu_{2}>0$ and the period of the periodic solutions increases as $\mu$ increases due to $\tau_{2}>0$.

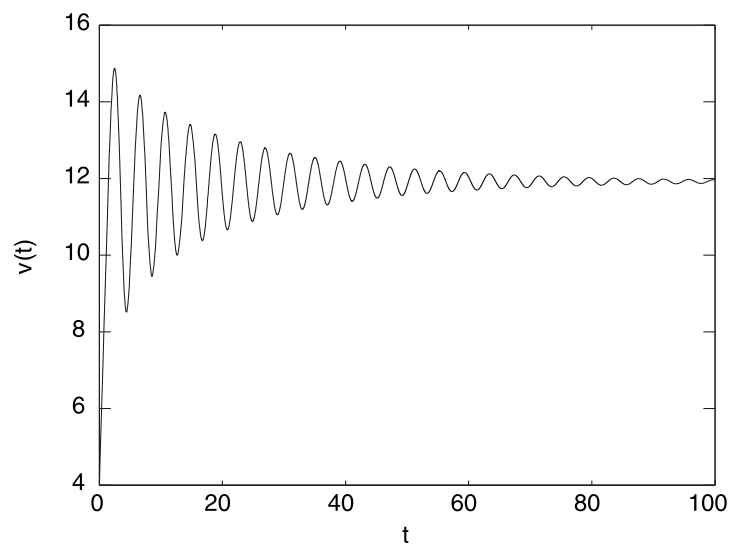

Now we choose appropriate value of $\alpha$ to control the Hopf bifurcation. It is easy to see from Theorem 7 that for a appropriate value of $\alpha$, we can delay the onset of the Hopf bifurcation. For example, by choosing

$\alpha=0.6$,

we can apply Theorem 7 and (22) in Sect. 3 to obtain

$\mu^{*}=\mu_{0}^{+}=0.1561, \quad \omega_{0}^{+}=1.8798$,

and

$\mu_{2}=0.0431, \quad \tau_{2}=-0.0140, \quad \beta_{2}=-0.2256$.

Note that the controlled small-world network model (6) has the same equilibrium point as that of the original small-world network model (2), but the critical value $\mu^{*}$ increases from 0.0408 to 0.1561 , implying that the onset of the Hopf bifurcation is delayed.

We choose $\mu=0.14, \alpha=0.6\left((\mu, \alpha) \in S_{1}\right.$, the same value of $\mu$ used in Fig. 6). With these parameters, $\tau_{0}=1.0806$ is obtained from Lemma 4 . Hence, by Theorem 5, instead of having a Hopf bifurcation, the controlled Internet congestion model (6) converges to the equilibrium point $V^{*}$ when $\tau=1 \in\left[0, \tau_{0}\right)$, as shown in Fig. 7.

We choose $\mu=0.16, \alpha=0.6\left((\mu, \alpha) \in S_{1}\right)$. With these parameters, $\tau_{0}=0.9830$ is obtained from Lemma 4. Hence, by Theorem 5, the equilibrium point $V^{*}$ is unstable when $\tau=1 \in\left(\tau_{0}, \infty\right)$, as shown in Fig. 8. Moreover, when $\mu=0.2, \alpha=0.6$ $\left((\mu, \alpha) \in S_{1}\right)$, we can derive $\tau_{0}=0.8426$ from Lem-

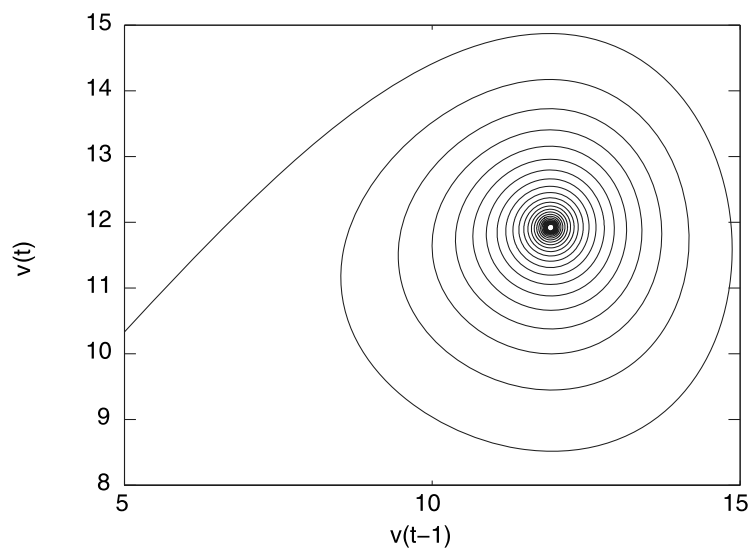

Fig. 4 Waveform plot and phase portrait of model (2) with $\mu=0.035$ 

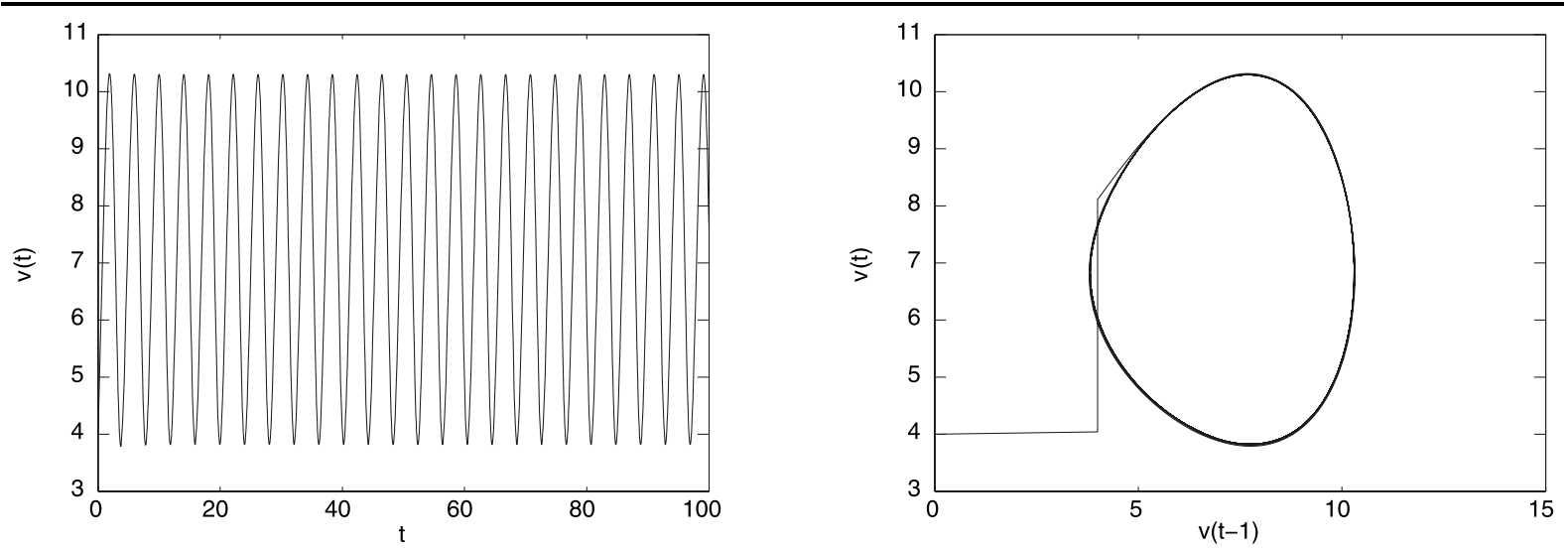

Fig. 5 Waveform plot and phase portrait of model (2) with $\mu=0.06$
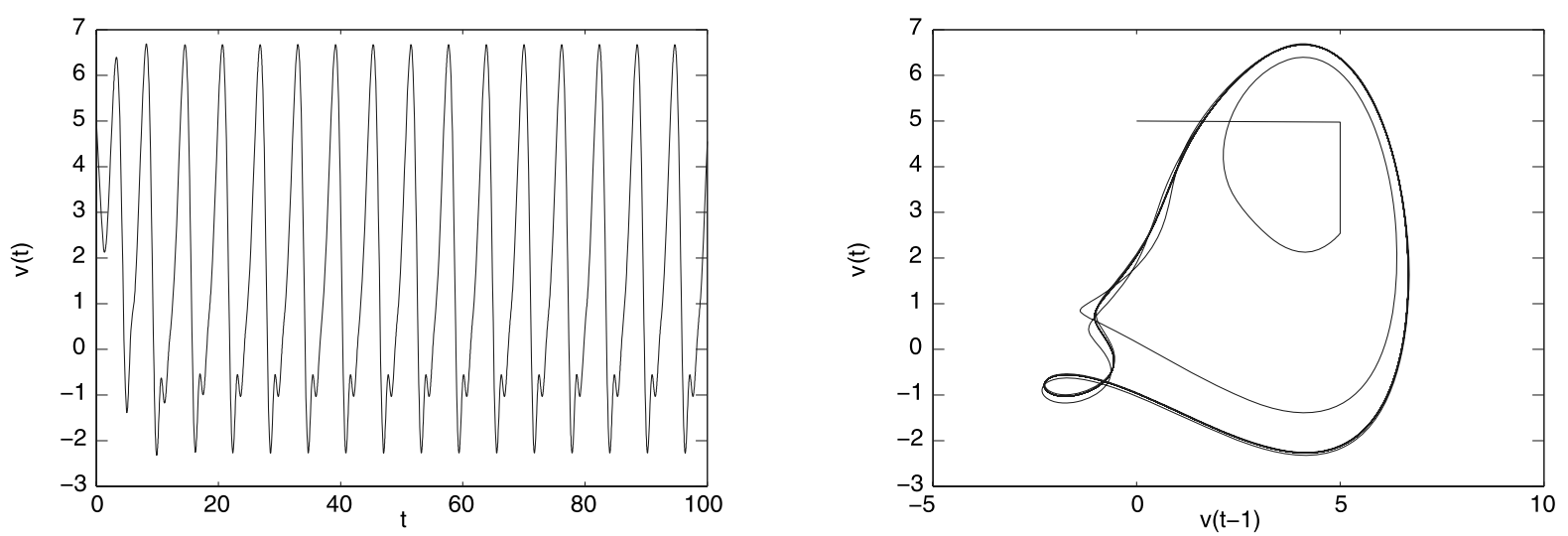

Fig. 6 Waveform plot and phase portrait of model (2) with $\mu=0.14$
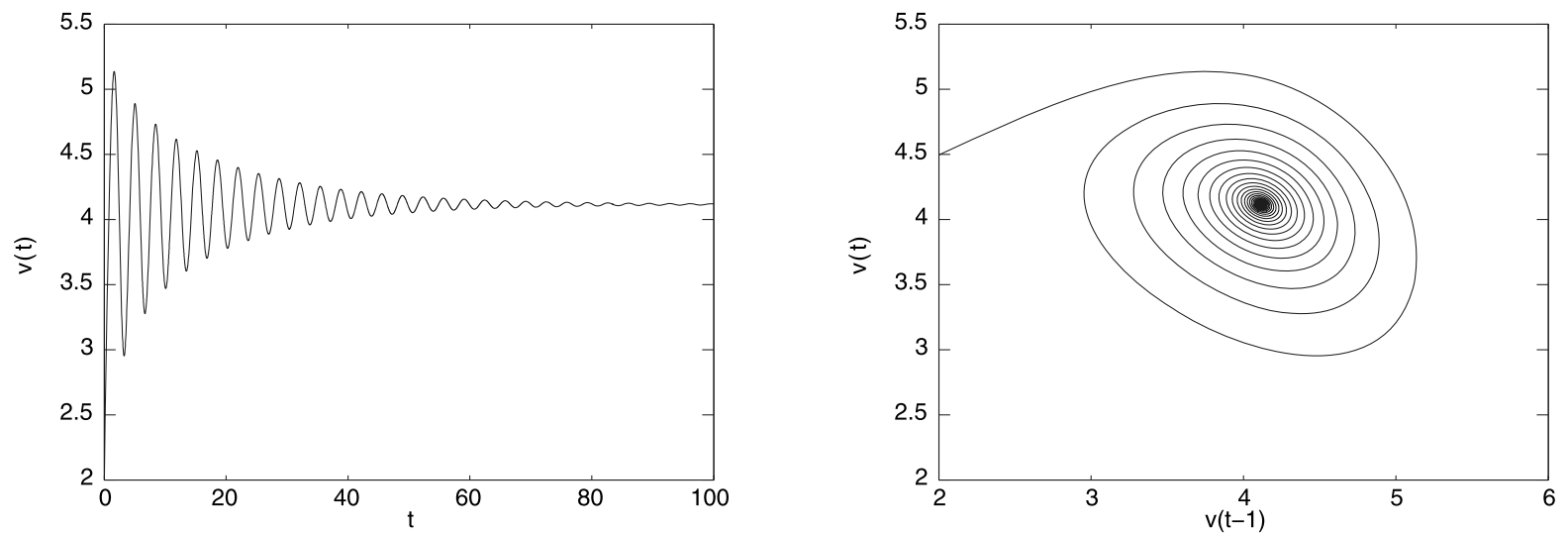

Fig. 7 Waveform plot and phase portrait of model (6) with $\mu=0.14$ and $\alpha=0.6$

ma 4. Therefore, by Theorem 5, the equilibrium point $V^{*}$ is also unstable when $\tau=1 \in\left(\tau_{0}, \infty\right)$, as shown in Fig. 9.
It is shown that when $\mu$ passes the critical value $\mu^{*}=0.1561$, a Hopf bifurcation occurs (see Figs. 8 and 9). The periodic orbits are stable since $\beta_{2}<0$. 

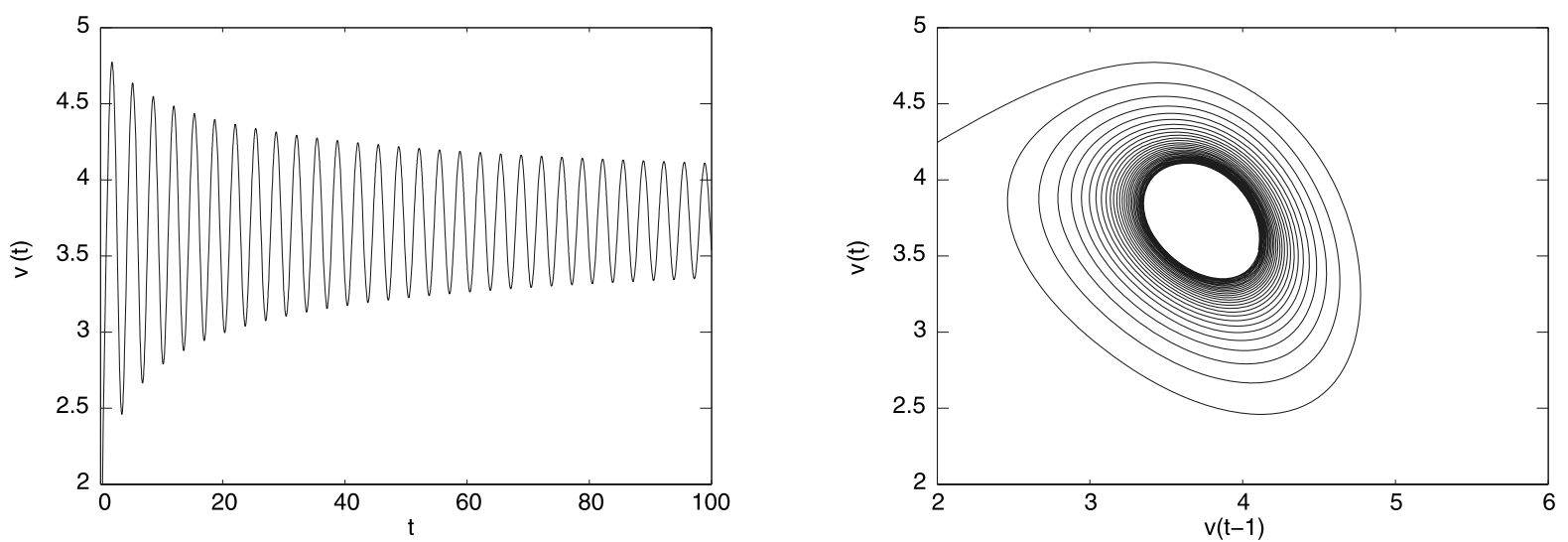

Fig. 8 Waveform plot and phase portrait of model (6) with $\mu=0.16$ and $\alpha=0.6$
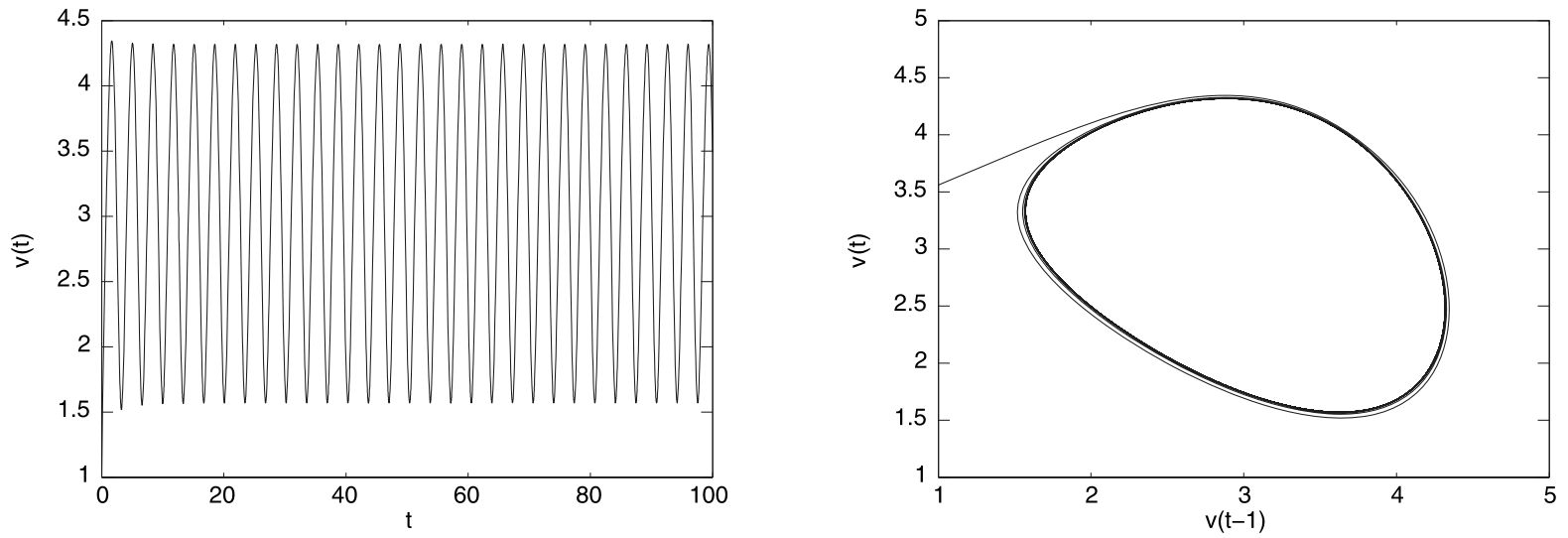

Fig. 9 Waveform plot and phase portrait of model (6) with $\mu=0.2$ and $\alpha=0.6$

Since $\mu_{2}>0$, the bifurcating periodic solutions exist at least for the value $\mu$ slightly larger than the critical value $\mu^{*}$. Since $\tau_{2}<0$, the period of the periodic solutions decreases as $\mu$ increases.

It can be shown that if we choose a larger value of $\alpha$, the small-world network model may not have a Hopf bifurcation even for larger values of $\mu$ (see Fig. 11). This indicates that the time-delayed feedback controller can delay the onset of Hopf bifurcation, thus guarantee a stationary total influenced volume for larger values of $\mu$. For example, when choosing $\alpha=2$, the controlled small-world network model (6) converges to the equilibrium solution $V^{*}$ if $\mu<\mu^{*}=0.6777$. In detail, we choose $\mu=0.5, \alpha=2$ $\left((\mu, \alpha) \in S_{1}\right)$. With these parameters, $\tau_{0}=2.0649$ is obtained from Lemma 4 . Hence, by Theorem 5, the controlled small-world network model (6) converges to the equilibrium point $V^{*}$ when $\tau=1 \in\left[0, \tau_{0}\right)$, as shown in Fig. 10.

Figure 12 displays a bifurcation diagram in terms of the parameter $\mu$ for model (6).

\section{Extended time-delayed feedback}

In this section, we consider a more general (extended) version of the delayed feedback control that employs a different delay from $\tau$ in uncontrolled system (2), which then becomes

$$
\begin{aligned}
\frac{\mathrm{d} V}{\mathrm{~d} t}= & \xi+V(t-\tau)-\mu \xi V^{2}(t-\tau) \\
& +\alpha\left(V\left(t-\tau_{1}\right)-V(t)\right) .
\end{aligned}
$$

Here we will discuss how time delay $\tau_{1}$ changes the domain of stability and the onset of Hopf bifurcation. 

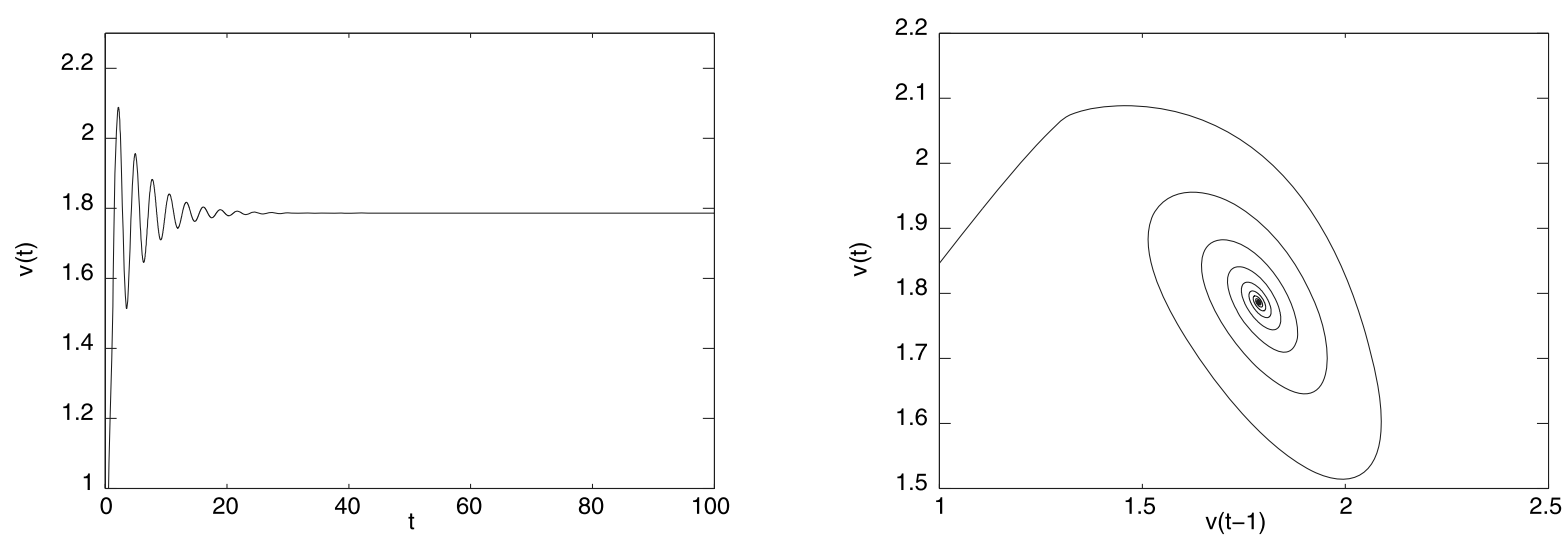

Fig. 10 Waveform plot and phase portrait of model (6) with $\mu=0.5$ and $\alpha=2$

Fig. 11 The fluctuation of $\mu^{*}$ depending on $\alpha$

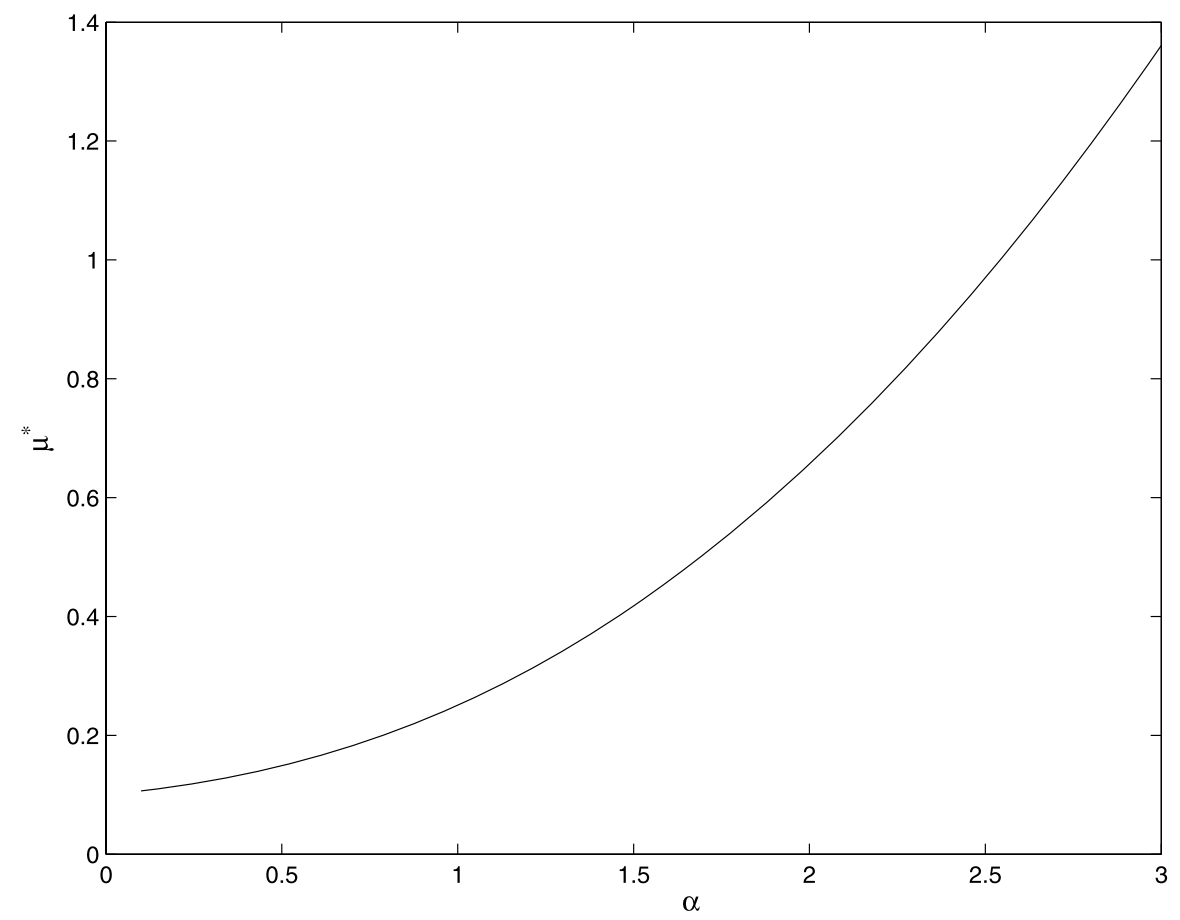

Let $x(t)=V(t)-V^{*}$. Linearizing the system (23) about the equilibrium point $V^{*}$, we can obtain

$\dot{x}(t)=-\alpha x(t)-\sqrt{1+4 \mu \xi^{2}} x(t-\tau)+\alpha x\left(t-\tau_{1}\right)$.

Then the characteristic equation of system (24) is

$\lambda+\alpha+\sqrt{1+4 \mu \xi^{2}} e^{-\lambda \tau}-\alpha e^{-\lambda \tau_{1}}=0$.
Theorem 9 When the parameter $\mu$ passes through the critical value $\mu=\mu^{*}$, where

$$
\mu^{*}=\left[\left(\frac{\alpha \cos \left(\omega_{0} \tau_{1}\right)-\alpha}{\cos \left(\omega_{0} \tau\right)}\right)^{2}-1\right] /\left(4 \xi^{2}\right),
$$

here $\omega_{0}$ is the root of

$$
\omega_{0}=\alpha \tan \left(\omega_{0} \tau\right)\left[\cos \left(\omega_{0} \tau_{1}\right)-1\right]-\alpha \sin \left(\omega_{0} \tau_{1}\right) .
$$


Fig. 12 Bifurcation diagram of model (2) without and with control
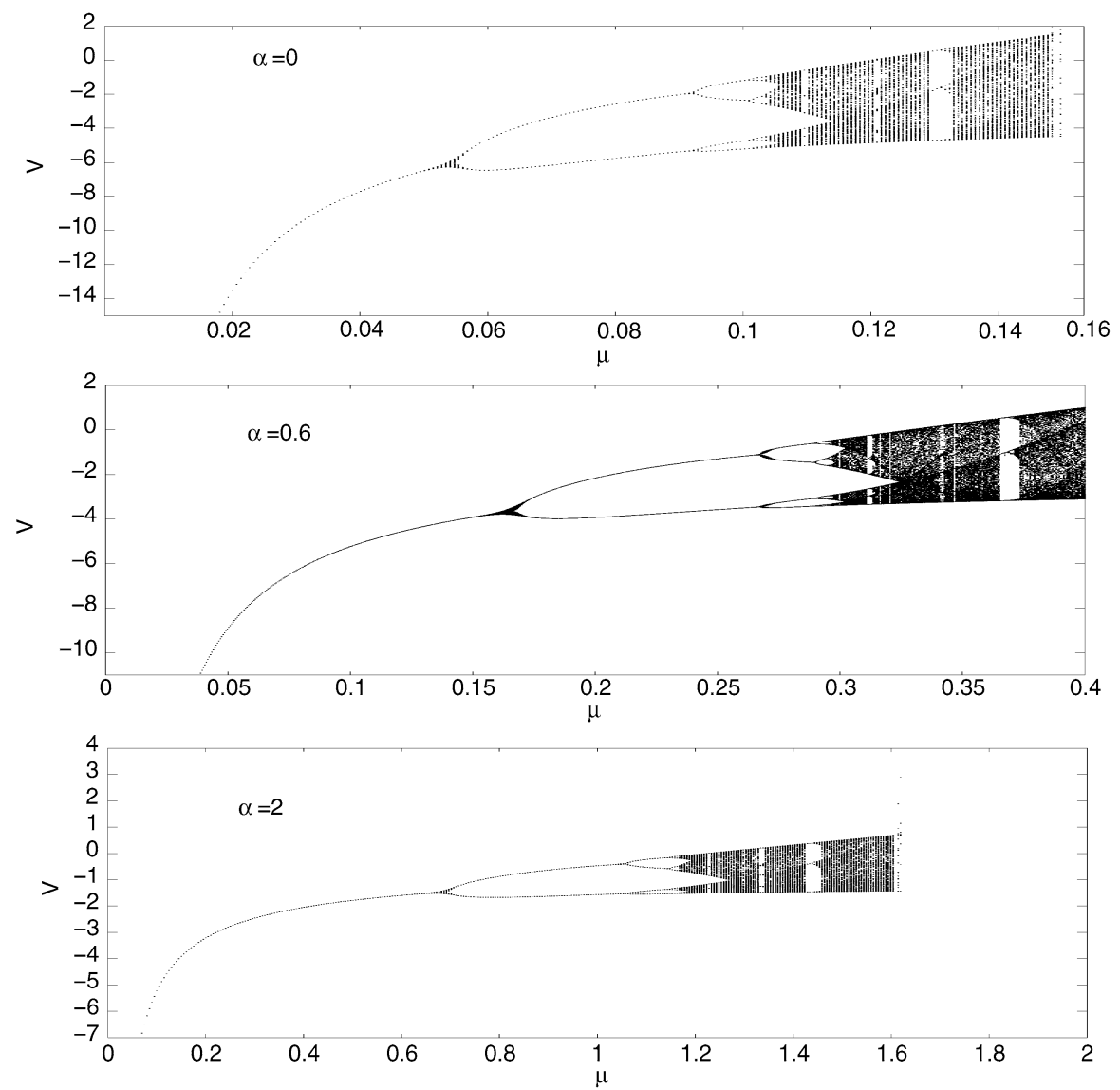

If

$2 \tau \xi^{2}-\frac{2 \alpha \tau_{1} \xi^{2}}{\sqrt{1+4 \mu^{*} \xi^{2}}} \cos \left[\omega_{0}\left(\tau-\tau_{1}\right)\right] \neq 0$

is satisfied, there is a Hopf bifurcation from the equilibrium $V^{*}$ to a periodic orbit in the controlled system (23).

Proof Suppose (25) has a pure imaginary solution $\lambda=$ $\mathrm{i} \omega_{0}\left(\omega_{0}>0\right)$, for some parameter value $\mu=\mu^{*}$. This leads to the following equation:

$\mathrm{i} \omega_{0}+\alpha+\sqrt{1+4 \mu^{*} \xi^{2}} e^{-\mathrm{i} \omega_{0} \tau}-\alpha e^{-\mathrm{i} \omega_{0} \tau_{1}}=0$,

which can be rewritten as

$\left\{\begin{array}{l}\alpha+\sqrt{1+4 \mu^{*} \xi^{2}} \cos \left(\omega_{0} \tau\right)-\alpha \cos \left(\omega_{0} \tau_{1}\right)=0, \\ \omega-\sqrt{1+4 \mu^{*} \xi^{2}} \sin \left(\omega_{0} \tau\right)+\alpha \sin \left(\omega_{0} \tau_{1}\right)=0 .\end{array}\right.$
So,

$\left\{\begin{array}{l}\mu^{*}=\left[\left(\frac{\alpha \cos \left(\omega_{0} \tau_{1}\right)-\alpha}{\cos \left(\omega_{0} \tau\right)}\right)^{2}-1\right] /\left(4 \xi^{2}\right), \\ \omega_{0}=\tan \left(\omega_{0} \tau\right)\left[\alpha \cos \left(\omega_{0} \tau_{1}\right)-\alpha\right]-\alpha \sin \left(\omega_{0} \tau_{1}\right) .\end{array}\right.$

The last condition for the occurrence of a Hopf bifurcation is $\frac{\mathrm{d}}{\mathrm{d} \mu}[\operatorname{Re} \lambda]_{\mu=\mu^{*}} \neq 0$. In the following, we will show that this condition is also satisfied.

Letting $\lambda=\rho+\mathrm{i} \omega$ and using (25), we have

$$
\left\{\begin{array}{c}
\rho+\alpha+\sqrt{1+4 \mu \xi^{2}} e^{-\rho \tau} \cos (\omega \tau) \\
-\alpha e^{-\rho \tau_{1}} \cos \left(\omega \tau_{1}\right)=0 \\
\omega-\sqrt{1+4 \mu \xi^{2}} e^{-\rho \tau} \sin (\omega \tau) \\
+\alpha e^{-\rho \tau_{1}} \sin \left(\omega \tau_{1}\right)=0
\end{array}\right.
$$

Hence, we have

$$
\frac{\mathrm{d}}{\mathrm{d} \mu}[\operatorname{Re} \lambda]_{\mu=\mu^{*}}=\left.\frac{\mathrm{d} \rho}{\mathrm{d} \mu}\right|_{\mu=\mu^{*}}
$$


Fig. 13 The fluctuation of $\mu^{*}$ depending on $\alpha$ for $\xi=3$ and $\tau=1$ as given by controlled system (23)

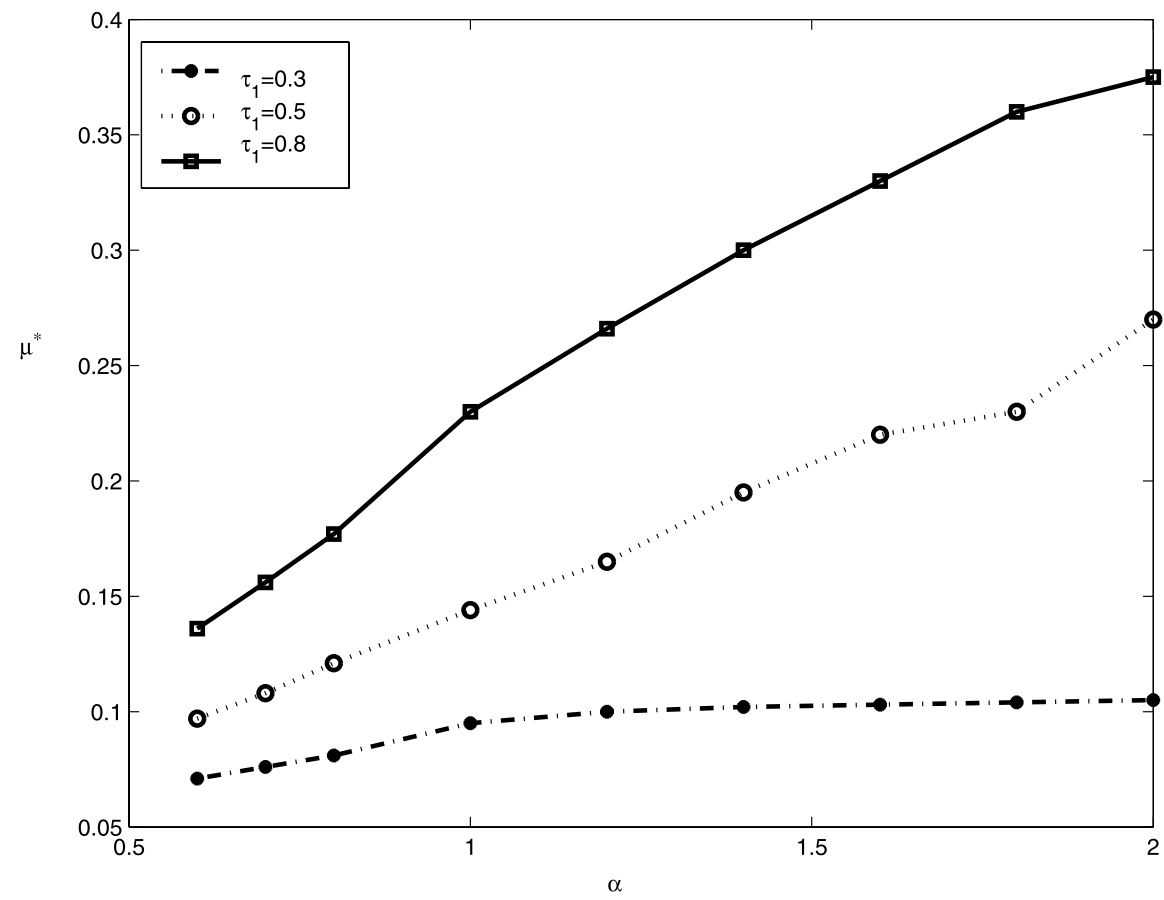

$$
=\frac{2 \tau \xi^{2}-\frac{2 \alpha \tau_{1} \xi^{2}}{\sqrt{1+4 \mu^{*} \xi^{2}}} \cos \left[\omega_{0}\left(\tau-\tau_{1}\right)\right]}{P^{2}+Q^{2}}
$$

where

$$
\begin{aligned}
& P=1-\tau \sqrt{1+4 \mu^{*} \xi^{2}} \cos \left(\omega_{0} \tau\right)+\alpha \tau_{1} \cos \left(\omega_{0} \tau_{1}\right), \\
& Q=\tau \sqrt{1+4 \mu^{*} \xi^{2}} \sin \left(\omega_{0} \tau\right)-\alpha \tau_{1} \sin \left(\omega_{0} \tau_{1}\right) .
\end{aligned}
$$

This implies that the parameter $\mu$ passes through the critical value $\mu^{*}$, there is Hopf bifurcation from the equilibrium $V^{*}$ to a periodic orbit.

Remark 5 Different from the time-delayed feedback discussed in Sect. 3, extended time-delayed feedback has a different delay $\tau_{1}$ from $\tau$ in uncontrolled system (2), which expands the regulated parameters besides feedback gain parameter $\alpha$ for bifurcation control. Thus, bifurcation control can be realized more conveniently in implementation of small-world networks.

Figure 13 displays the dependence of $\mu^{*}$ upon the feedback gain $\alpha$ according to controlled system (23) for $\xi=3$ and $\tau=1$. The solid curve corresponds to a delay of $\tau_{1}=0.8$, the dotted curve to $\tau_{1}=0.5$, the dash-dotted curve to $\tau_{1}=0.3$. The values of the $\mu^{*}$ are calculated by solving (28) numerically. For increasing the feedback gain $\alpha$, the critical value $\mu^{*}$ increases for a fixed time delay $\tau_{1}$. Increasing $\alpha$ postpones the onset of Hopf bifurcation and reduces the instability. Hence the control is successful. It can be seen that increasing time delay of $\tau_{1}$ raises the value of $\mu^{*}$ to a fixed feedback gain $\alpha$. The degree on postponement of $\mu^{*}$ depends on the delay $\tau_{1}$. Further increase of $\tau_{1}$ elevates the value of $\mu^{*}$ for a fixed feedback gain $\alpha$.

In the case of a combination of $\xi=3, \tau=1$ and $\alpha=0.6$, the values $0.0713,0.0971$ and 0.1362 of $\mu^{*}$ correspond to $\tau_{1}=0.3,0.5$ and 0.8 , respectively. Panel (a) of Fig. $14\left(\tau_{1}=0.3\right)$ shows $V(t)$ tends to the equilibrium $V^{*}$ at $\mu=0.065$ for controlled system (23), while panel (b) of Fig. 14 depicts the equilibrium $V^{*}$ is unstable at $\mu=0.09$. A Hopf bifurcation emerges when $\mu$ passes through the $\mu^{*}=0.0713$. The position of original bifurcation point is postponed (see Figs. 4 and 5). Panel (a) of Fig. $15\left(\tau_{1}=0.5\right)$ displays that the controlled system (23) converges to the equilibrium $V^{*}$ again at the same value 0.09 of $\mu$ used in panel (b) of Fig. 14. If $\mu=0.13>\mu^{*}=0.0971$, the equilibrium $V^{*}$ becomes unstable (see panel (b) of Fig. 15). It can be shown that if one chooses larger value of $\tau_{1}$, the controlled small-world network 

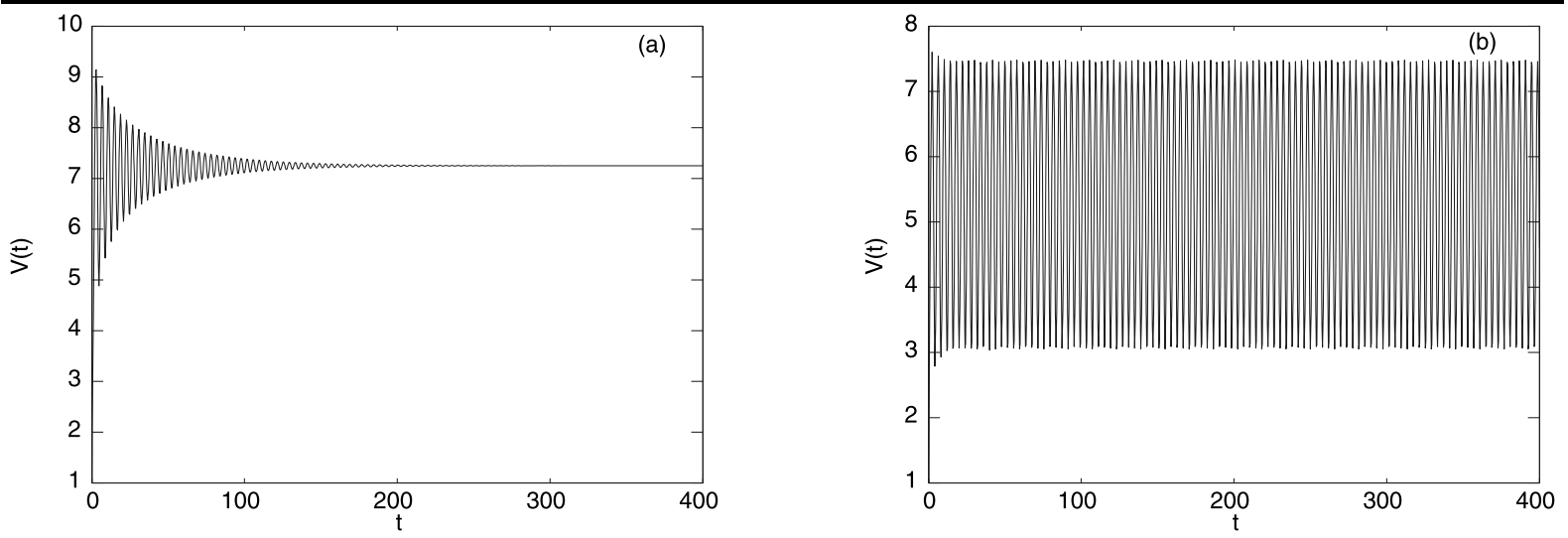

Fig. 14 Waveform plot of model (23) with $\tau_{1}=0.3$ and $\alpha=0.6$ (panel (a): $\mu=0.065$; panel (b): $\left.\mu=0.09\right)$
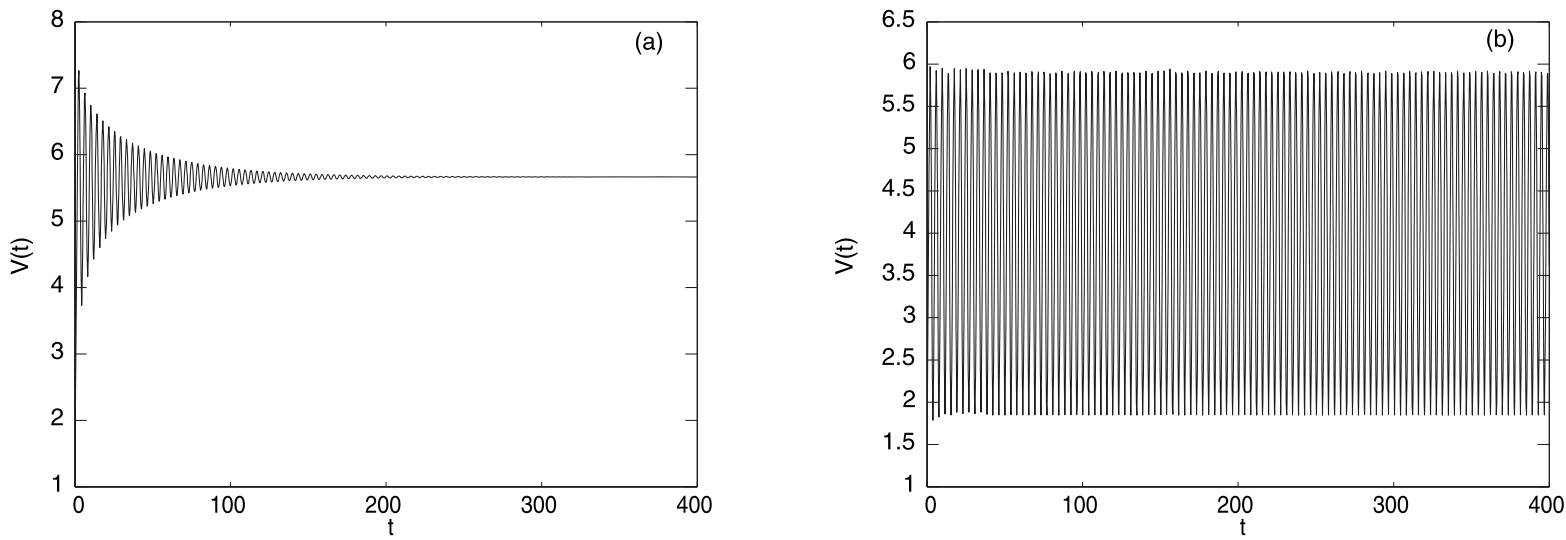

Fig. 15 Waveform plot of model (23) with $\tau_{1}=0.5$ and $\alpha=0.6(\operatorname{panel}(\mathbf{a}): \mu=0.09$; panel $(\mathbf{b}): \mu=0.13)$

model (23) may not have a Hopf bifurcation even for larger values of $\mu$ (see Fig. 16).

Figure 17 displays a bifurcation diagram in terms of the parameter $\mu$ for model (23).

\section{Latency time effects}

This section is focused on nonzero latency times, which can be associated with the generation and injection of feedback signal [25]. It has been shown experimentally [26] in the case of an unstable periodic orbit that latency can have important effects on the controllability of the system and might limit the success of the time-delayed feedback. A theoretical explanation can be found in $[27,28]$. Here we will study how latency times change the domain of control in Hopf bifurcation.

The latency time $\delta$ can be included as an additional time delay in the control force of (6), which then be- comes

$F(t-\delta)=\alpha(V(t-\tau-\delta)-V(t-\delta))$,

leading to a characteristic equation similar to (9) but with two additional exponential factors

$\lambda+\sqrt{1+4 \mu \xi^{2}} e^{-\lambda \tau}+\alpha e^{-\lambda \delta}-\alpha e^{-\lambda(\tau+\delta)}=0$,

or, separating into real and imaginary parts,

$$
\left\{\begin{array}{l}
\rho+\sqrt{1+4 \mu \xi^{2}} e^{-\rho \tau} \cos (\omega \tau)+\alpha e^{-\rho \delta} \cos (\omega \delta) \\
\quad-\alpha e^{-\rho(\tau+\delta)} \cos [\omega(\tau+\delta)]=0 \\
\omega-\sqrt{1+4 \mu \xi^{2}} e^{-\rho \tau} \sin (\omega \tau)-\alpha e^{-\rho \delta} \sin (\omega \delta) \\
+\alpha e^{-\rho(\tau+\delta)} \sin [\omega(\tau+\delta)]=0
\end{array}\right.
$$

where $\rho$ and $\omega$ denote the real and imaginary part of $\lambda$, respectively. 

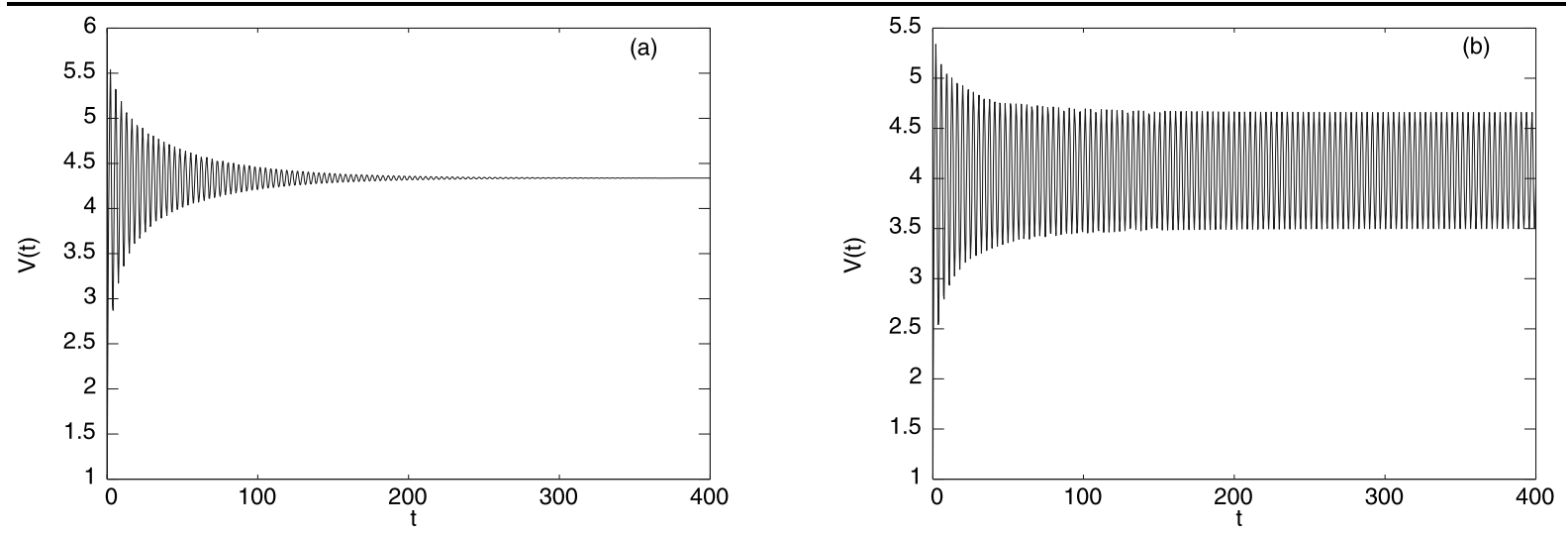

Fig. 16 Waveform plot of model (23) with $\tau_{1}=0.8$ and $\alpha=0.6$ (panel (a): $\mu=0.13$; panel (b): $\left.\mu=0.14\right)$

Fig. 17 Bifurcation diagram of system (23) with control

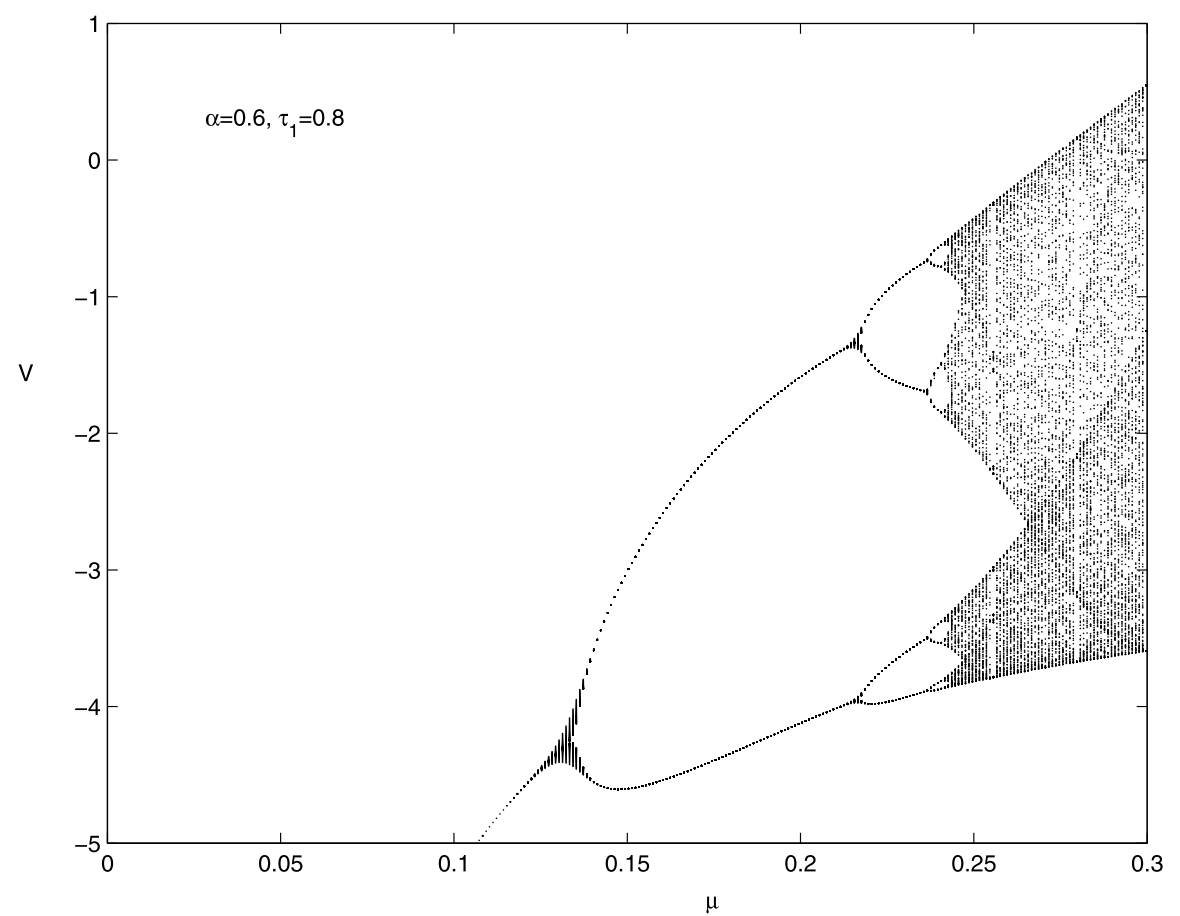

Theorem 10 When the parameter $\mu$ passes through the critical value $\mu=\mu^{*}$, where

$\mu^{*}=\left\{\left[\frac{\alpha \cos \left[\omega_{0}(\tau+\delta)\right]-\alpha \cos \left(\omega_{0} \delta\right)}{\cos \left(\omega_{0} \tau\right)}\right]^{2}-1\right\} /\left(4 \xi^{2}\right)$,

here $\omega_{0}$ is the root of

$$
\begin{gathered}
\omega_{0}=\alpha \tan \left(\omega_{0} \tau\right)\left\{\cos \left[\omega_{0}(\tau+\delta)\right]-\cos \left(\omega_{0} \delta\right)\right\} \\
+\alpha \sin \left(\omega_{0} \delta\right)-\alpha \sin \left[\omega_{0}(\tau+\delta)\right] .
\end{gathered}
$$

If

$$
\begin{aligned}
& \frac{2 \xi^{2}}{\sqrt{1+4 \mu^{*} \xi^{2}}}\left\{\tau \sqrt{1+4 \mu^{*} \xi^{2}} \cos \left(2 \omega_{0} \tau\right)-\cos \left(\omega_{0} \tau\right)\right. \\
& +\alpha \delta \cos \left[\omega_{0}(\tau+\delta)\right] \\
& \left.-\alpha(\tau+\delta) \cos \left[\omega_{0}(2 \tau+\delta)\right]\right\} \neq 0
\end{aligned}
$$

is satisfied, there is a Hopf bifurcation from the equilibrium $V^{*}$ to a periodic orbit for the small-world network model with control (32). 
Proof Suppose (33) has a pure imaginary solution $\lambda=$ $\mathrm{i} \omega_{0}\left(\omega_{0}>0\right)$, for some parameter value $\mu=\mu^{*}$. This leads to the following equation:

$$
\begin{aligned}
& \mathrm{i} \omega_{0}+\sqrt{1+4 \mu \xi^{2}} e^{-\mathrm{i} \omega_{0} \tau}+\alpha e^{-\mathrm{i} \omega_{0} \delta}-\alpha e^{-\mathrm{i} \omega_{0}(\tau+\delta)} \\
& \quad=0
\end{aligned}
$$

which can be rewritten as

$$
\left\{\begin{array}{l}
\sqrt{1+4 \mu \xi^{2}} \cos \left(\omega_{0} \tau\right)+\alpha \cos \left(\omega_{0} \delta\right) \\
\quad-\alpha \cos \left[\omega_{0}(\tau+\delta)\right]=0 \\
\omega_{0}-\sqrt{1+4 \mu \xi^{2}} \sin \left(\omega_{0} \tau\right)-\alpha \sin \left(\omega_{0} \delta\right) \\
+\alpha \sin \left[\omega_{0}(\tau+\delta)\right]=0
\end{array}\right.
$$

So,

$$
\left\{\begin{aligned}
\mu^{*}= & \left\{\left[\frac{\alpha \cos \left[\omega_{0}(\tau+\delta)\right]-\alpha \cos \left(\omega_{0} \delta\right)}{\cos \left(\omega_{0} \tau\right)}\right]^{2}\right. \\
& -1\} /\left(4 \xi^{2}\right), \\
\omega_{0}= & \alpha \tan \left(\omega_{0} \tau\right)\left\{\cos \left[\omega_{0}(\tau+\delta)\right]-\cos \left(\omega_{0} \delta\right)\right\} \\
& +\alpha \sin \left(\omega_{0} \delta\right)-\alpha \sin \left[\omega_{0}(\tau+\delta)\right] .
\end{aligned}\right.
$$

$$
\begin{aligned}
& \frac{\mathrm{d}}{\mathrm{d} \mu}[\operatorname{Re} \lambda]_{\mu=\mu^{*}} \\
& \quad=\left.\frac{\mathrm{d} \rho}{\mathrm{d} \mu}\right|_{\mu=\mu^{*}} \\
& =\frac{\frac{2 \xi^{2}}{\sqrt{1+4 \mu^{*} \xi^{2}}}\left\{\tau \sqrt{1+4 \mu^{*} \xi^{2}} \cos \left(2 \omega_{0} \tau\right)-\cos \left(\omega_{0} \tau\right)+\alpha \delta \cos \left[\omega_{0}(\tau+\delta)\right]-\alpha(\tau+\delta) \cos \left[\omega_{0}(2 \tau+\delta)\right]\right\}}{M^{2}+N^{2}} \neq 0
\end{aligned}
$$

where

$$
\begin{aligned}
M= & 1-\tau \sqrt{1+4 \mu^{*} \xi^{2}} \cos \left(\omega_{0} \tau\right)-\alpha \delta \cos \left(\omega_{0} \delta\right) \\
& +\alpha(\tau+\delta) \cos \left[\omega_{0}(\tau+\delta)\right], \\
N= & \tau \sqrt{1+4 \mu^{*} \xi^{2}} \sin \left(\omega_{0} \tau\right)+\alpha \delta \sin \left(\omega_{0} \delta\right) \\
& -\alpha(\tau+\delta) \sin \left[\omega_{0}(\tau+\delta)\right] .
\end{aligned}
$$

This implies that the parameter $\mu$ passes through the critical value $\mu^{*}$, there is Hopf bifurcation from the equilibrium $V^{*}$ to a periodic orbit.

Remark 6 It is shown in Theorem 10 that the goal of bifurcation control can be ensured if time-delayed feedback control (32) with latency time is carried out for uncontrolled model (2). Note that the $\mu^{*}$ is dependent on feedback gain parameter $\alpha$ and latency time $\delta$.
Therefore, the bifurcation control approach developed in this section is more efficient.

Figure 18 displays the dependence of $\mu^{*}$ upon the feedback gain $\alpha$ according to the small-world network model (2) with control (32) for $\xi=3$ and $\tau=1$. The solid curve corresponds to latency time of $\delta=0.1$, the dotted curve to $\delta=0.15$, the dash-dotted curve to $\delta=0.2$. The values of the $\mu^{*}$ are calculated by solving (37) numerically. For increasing the feedback gain $\alpha$, the critical value $\mu^{*}$ increases for a fixed latency time $\delta$. Increasing $\alpha$ postpones the onset of Hopf bifurcation and reduces the instability. Hence the control scheme is successful. The degree on postponement of $\mu^{*}$ depends on the latency time $\delta$. Further decrease of $\delta$ raises the value of $\mu^{*}$ for a fixed feedback gain $\alpha$.

In the case of a combination of $\xi=3, \tau=1$ and $\alpha=0.6$, the values $0.1471,0.1392$ and 0.1313 of 
Fig. 18 The fluctuation of $\mu^{*}$ depending on $\alpha$ for $\xi=3$ and $\tau=1$ as given by model (2) with control (32)
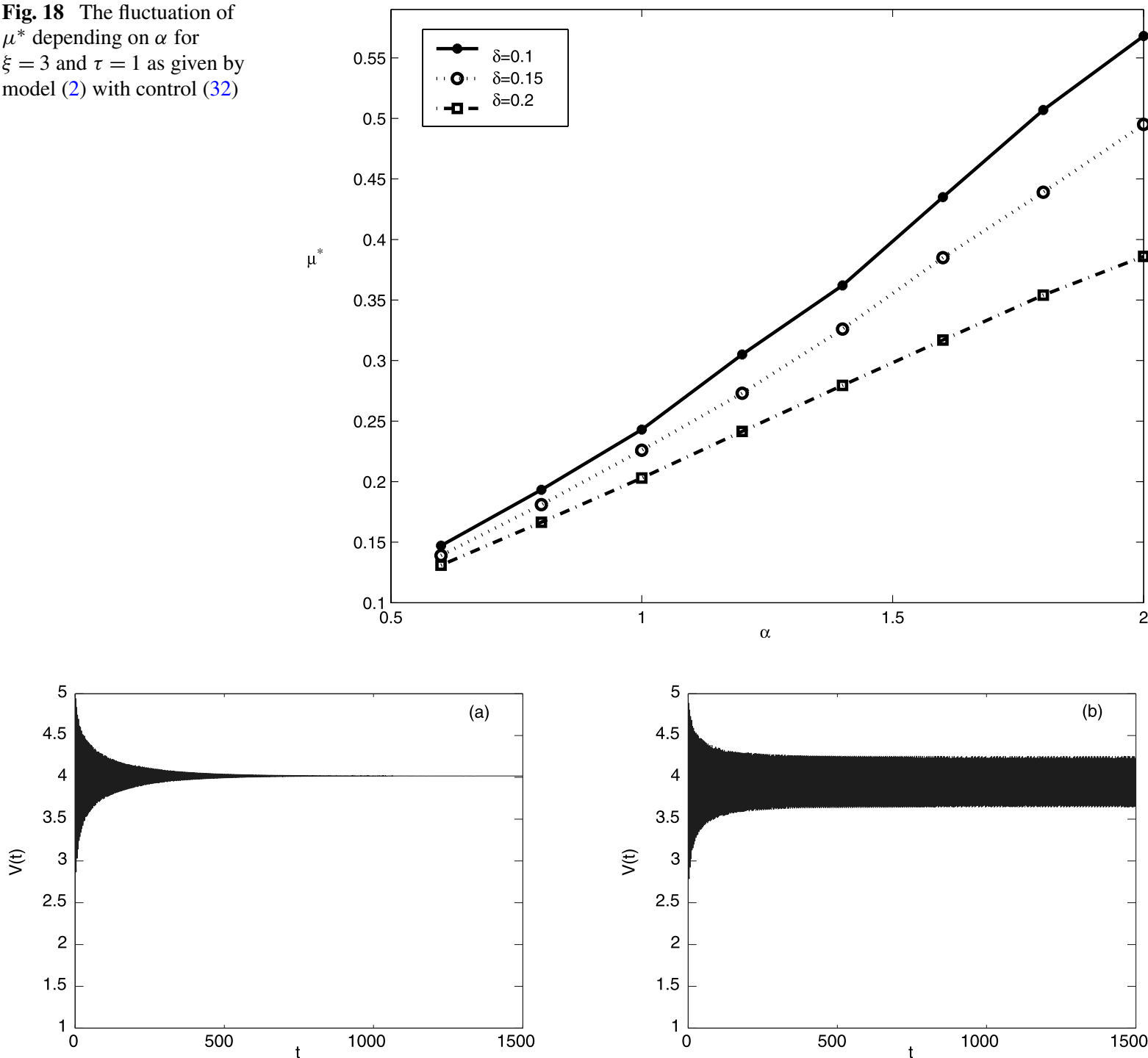

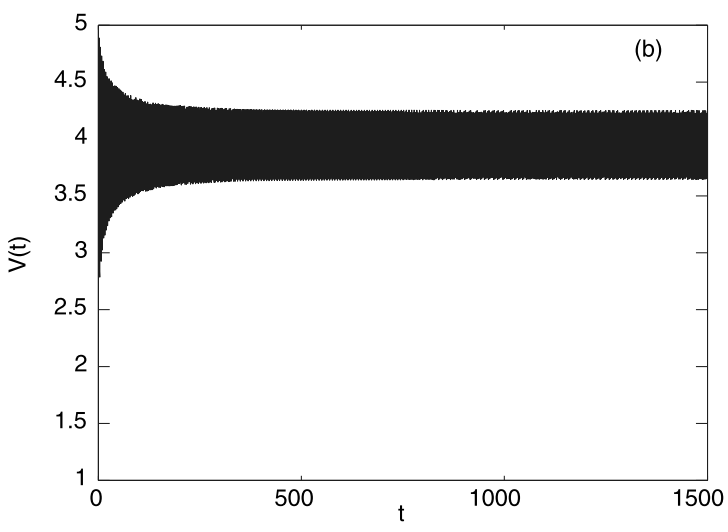

Fig. 19 Waveform plot of model (23) with $\delta=0.1$ and $\alpha=0.6$ (panel (a): $\mu=0.145$; panel (b): $\mu=0.148)$

$\mu^{*}$ correspond to $\delta=0.1,0.15$ and 0.2 , respectively. Panel (a) of Fig. 19 ( $\delta=0.1)$ shows trajectories converge to the equilibrium $V^{*}$ at $\mu=0.145$ for the model (2) with control (32), while panel (b) of Fig. 19 depicts the equilibrium $V^{*}$ is unstable at $\mu=0.148$. A Hopf bifurcation emerges when $\mu$ passes through the $\mu^{*}=0.1471$. Panel (b) of Fig. $20(\delta=0.15)$ displays that the equilibrium point $V^{*}$ loses stability again at the value 0.145 of $\mu$ used in panel (a) of Fig. 19. If $\mu=0.135<\mu^{*}=0.1392$, the equilibrium $V^{*}$ becomes stable (see panel (a) of Fig. 20). It can be shown that if one chooses larger value of $\delta$, the con- trolled small-world network model may have a Hopf bifurcation even for smaller values of $\mu$ (see Fig. 21).

Figure 22 displays a bifurcation diagram in terms of the parameter $\mu$ for model (2) under control (32).

\section{Low-pass filtering}

It has been found that high-frequency modulations of the control signal, due to additional high-frequency components in the signal besides the main frequency, can render the time-delayed auto-synchronization 

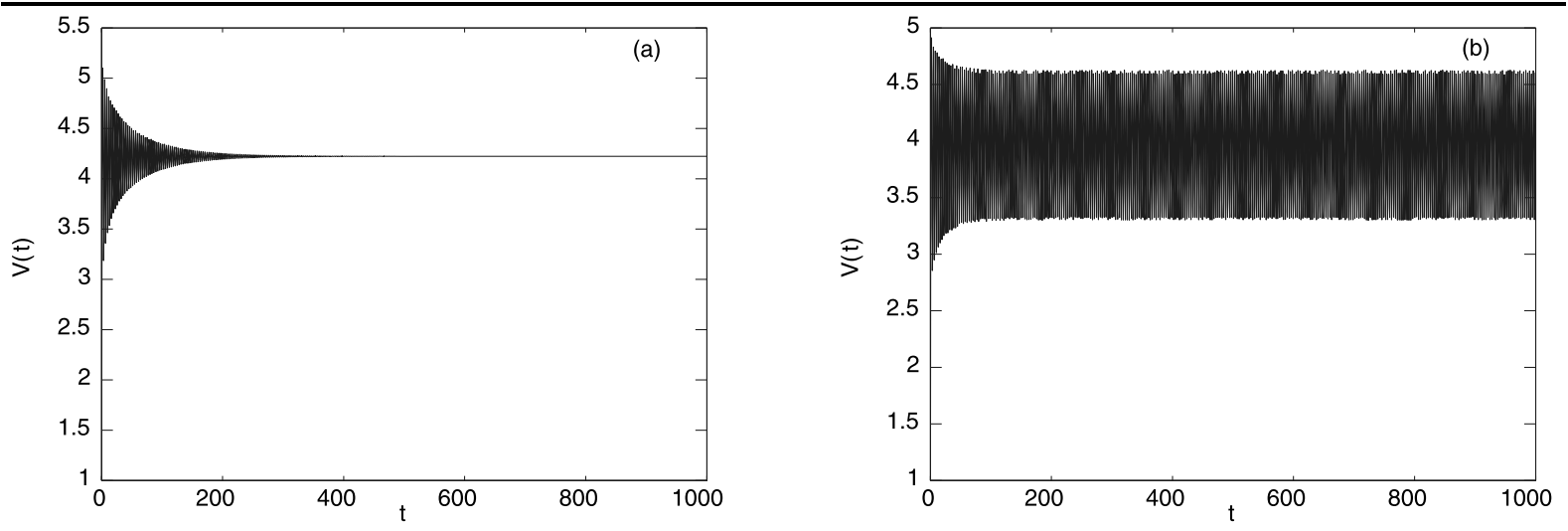

Fig. 20 Waveform plot of model (23) with $\delta=0.15$ and $\alpha=0.6(\operatorname{panel}(\mathbf{a}): \mu=0.135$; panel $(\mathbf{b}): \mu=0.145)$
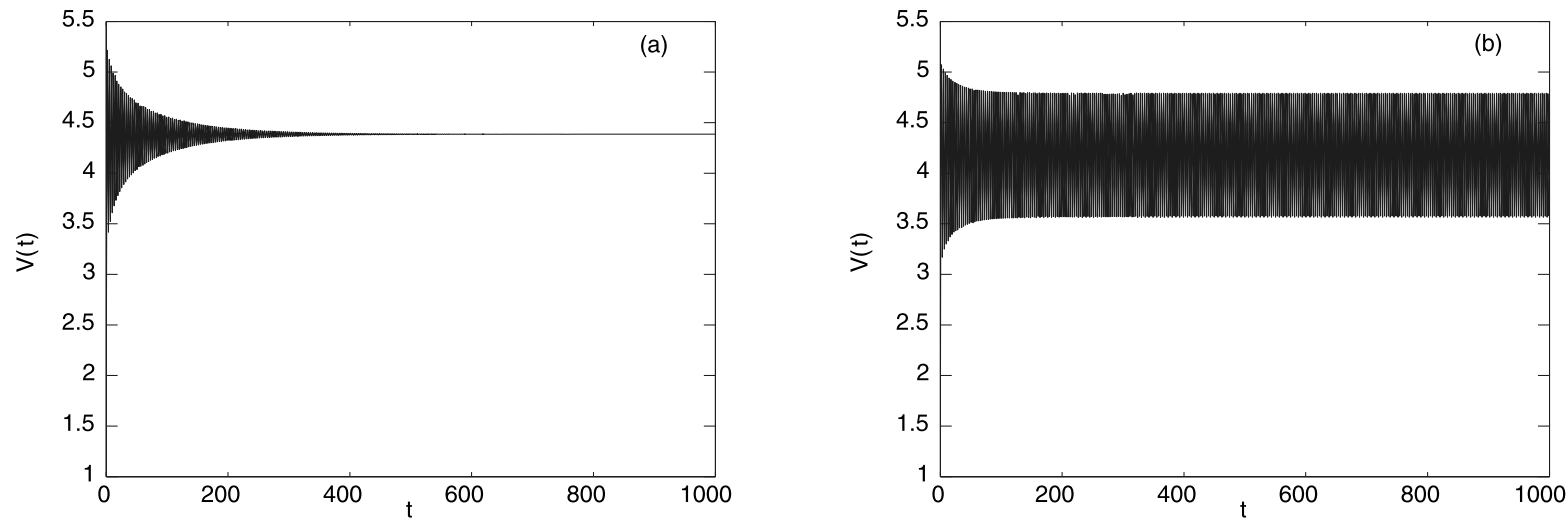

Fig. 21 Waveform plot of model (23) with $\delta=0.2$ and $\alpha=0.6$ (panel (a): $\mu=0.128$; panel (b): $\mu=0.135)$

(TDAS) control method unstable [29]. As shown in that work, an additional low-pass filter included in the control loop can overcome this limitation, and unstable periodic orbits can be stabilized [29]. On the other hand, in electronic signal processing the finite response time of the circuit often imposes unavoidable low-pass filtering, and its effect upon feedback control is not clear. In this section we will show that a low-pass filter changes domain of stability, and shifts the onset of Hopf bifurcation to larger values. Note that low-pass filtering has been successfully used to stabilize unstable steady states by generating a control force from the difference of the current state to its filtered counterpart [30].

The TDAS control force with an additional lowpass filter can be written as

$F(t)=\alpha(\bar{V}(t-\tau)-\bar{V}(t))$, where $\bar{V}$ denotes the filtered version of $V$ defined by

$\bar{V}(t)=\beta \int_{-\infty}^{t} V(\sigma) e^{-\beta(t-\sigma)} \mathrm{d} \sigma$,

with the cutoff frequency $\beta$. Equivalently, the convolution integrals can be replaced by one additional differential equations such that the original one-dimensional system becomes two-dimensional,

$\left\{\begin{aligned} \dot{V}(t)= & \xi+V(t-\tau)-\mu \xi V^{2}(t-\tau) \\ & +\alpha(\bar{V}(t-\tau)-\bar{V}(t)), \\ \dot{\bar{V}}(t)= & -\beta \bar{V}(t)+\beta V(t) .\end{aligned}\right.$

This system of differential equations yields a characteristic equation of the form 


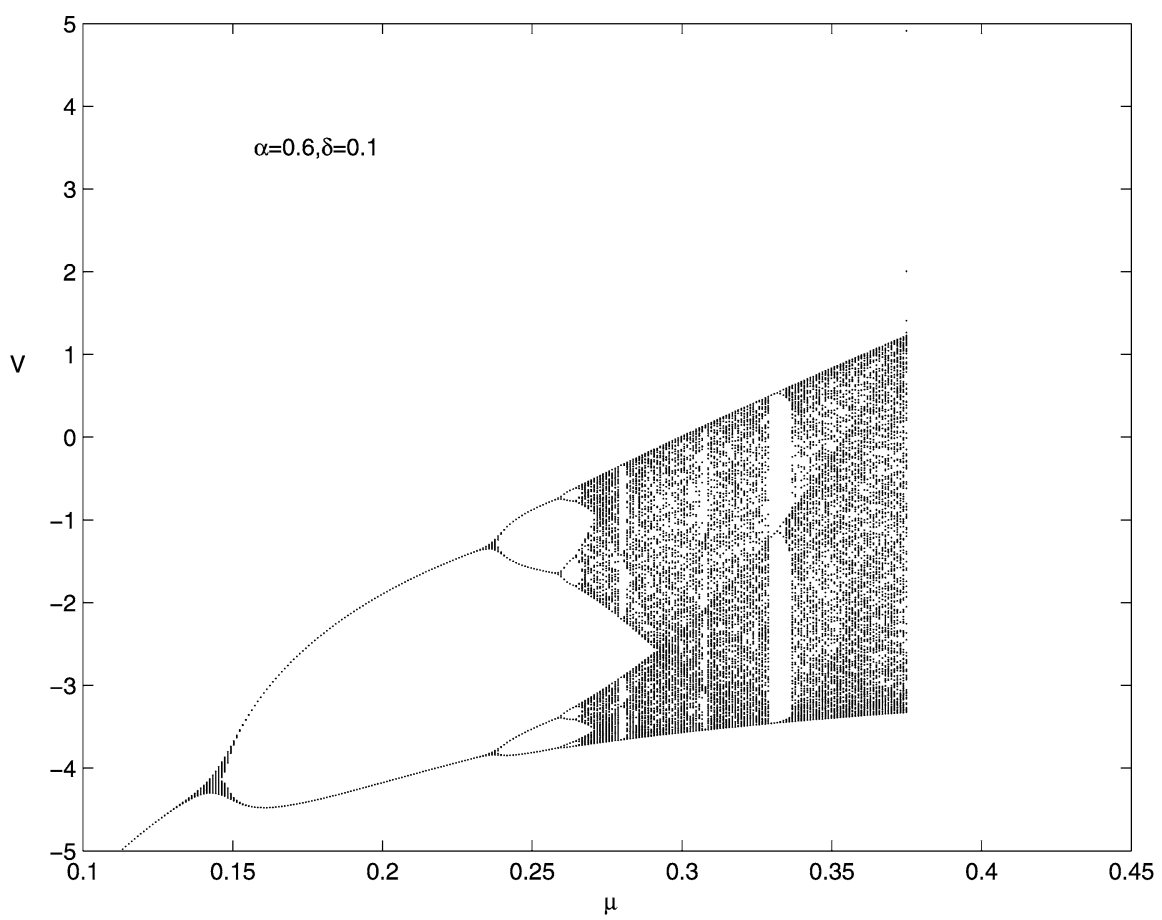

Fig. 22 Bifurcation diagram of model (2) with control (32)

$\operatorname{det}\left(\begin{array}{cc}\lambda+\sqrt{1+4 \mu \xi^{2}} e^{-\lambda \tau} & \alpha-\alpha e^{-\lambda \tau} \\ -\beta & \lambda+\beta\end{array}\right)=0$,

which can be written as

$$
\begin{aligned}
& \lambda^{2}+\beta \lambda+\alpha \beta+\left(\sqrt{1+4 \mu \xi^{2}} \lambda\right. \\
& \left.\quad+\beta \sqrt{1+4 \mu \xi^{2}}-\alpha \beta\right) e^{-\lambda \tau}=0,
\end{aligned}
$$

or, equivalently, using $\lambda=\rho+\mathrm{i} \omega$,

$$
\left\{\begin{array}{l}
\rho^{2}-\omega^{2}+\beta \rho+\alpha \beta+\omega e^{-\rho \tau} \sin (\omega \tau) \sqrt{1+4 \mu \xi^{2}} \\
\quad+e^{-\rho \tau} \cos (\omega \tau)\left[\rho \sqrt{1+4 \mu \xi^{2}}\right. \\
\left.+\beta \sqrt{1+4 \mu \xi^{2}}-\alpha \beta\right]=0 \\
2 \rho \omega+\beta \omega+\omega e^{-\rho \tau} \cos (\omega \tau) \sqrt{1+4 \mu \xi^{2}} \\
\quad-e^{-\rho \tau} \sin (\omega \tau)\left[\rho \sqrt{1+4 \mu \xi^{2}}\right. \\
\left.+\beta \sqrt{1+4 \mu \xi^{2}}-\alpha \beta\right]=0
\end{array}\right.
$$

Note that in the limit of large cutoff frequencies, i.e., $\beta \rightarrow \infty$, (44) reduces to the characteristic equation (9) of Sect. 3 .

Theorem 11 When the parameter $\mu$ passes through the critical value, $\mu=\mu^{*}$, where

$\mu^{*}=\left\{\left[\frac{\beta \omega_{0}+\alpha \beta \sin \left(\omega_{0} \tau\right)}{\omega \cos \left(\omega_{0} \tau\right)-\beta \sin \left(\omega_{0} \tau\right)}\right]^{2}-1\right\} /\left(4 \xi^{2}\right)$,

here $\omega_{0}$ is the root of

$$
\begin{aligned}
& \frac{\omega_{0} \sin \left(\omega_{0} \tau\right)+\beta \cos \left(\omega_{0} \tau\right)}{\omega \cos \left(\omega_{0} \tau\right)-\beta \sin \left(\omega_{0} \tau\right)} \\
& =-\frac{\omega_{0}^{2}-\alpha \beta+\alpha \beta \cos \left(\omega_{0} \tau\right)}{\beta \omega_{0}+\alpha \beta \sin \left(\omega_{0} \tau\right)} .
\end{aligned}
$$

If

$$
\begin{aligned}
& \frac{2 \xi^{2}}{\sqrt{1+4 \mu^{*} \xi^{2}}}\left[\beta \omega_{0} \sin \left(\omega_{0} \tau\right)-\left(\beta^{2}+2 \omega_{0}^{2}\right)\right. \\
& \left.\quad \times \cos \left(\omega_{0} \tau\right)-\alpha \tau \beta^{2}\right]+2 \xi^{2}\left(\tau \omega_{0}^{2}-\beta+\tau \beta^{2}\right) \neq 0
\end{aligned}
$$


is satisfied, there is a Hopf bifurcation from the equilibrium $V^{*}$ to a periodic orbit for the controlled system (42).

Proof Suppose (44) has a pure imaginary solution $\lambda=$ $\mathrm{i} \omega_{0}\left(\omega_{0}>0\right)$, for some parameter value $\mu=\mu^{*}$. This leads to the following equation:

$$
\begin{gathered}
-\omega_{0}^{2}+\mathrm{i} \beta \omega_{0}+\alpha \beta+\left(\mathrm{i} \sqrt{1+4 \mu \xi^{2}} \omega_{0}\right. \\
\left.+\beta \sqrt{1+4 \mu \xi^{2}}-\alpha \beta\right) e^{-\mathrm{i} \omega_{0} \tau}=0,
\end{gathered}
$$

which can be rewritten as

$$
\left\{\begin{array}{c}
-\omega_{0}^{2}+\alpha \beta+\omega_{0} \sin \left(\omega_{0} \tau\right) \sqrt{1+4 \mu \xi^{2}} \\
+\cos \left(\omega_{0} \tau\right)\left[\beta \sqrt{1+4 \mu \xi^{2}}-\alpha \beta\right]=0 \\
\beta \omega_{0}+\omega_{0} \cos \left(\omega_{0} \tau\right) \sqrt{1+4 \mu \xi^{2}} \\
-\sin \left(\omega_{0} \tau\right)\left[\beta \sqrt{1+4 \mu \xi^{2}}-\alpha \beta\right]=0 .
\end{array}\right.
$$

So,

$$
\left\{\begin{array}{l}
\mu^{*}=\left\{\left[\frac{\beta \omega_{0}+\alpha \beta \sin \left(\omega_{0} \tau\right)}{\omega \cos \left(\omega_{0} \tau\right)-\beta \sin \left(\omega_{0} \tau\right)}\right]^{2}-1\right\} /\left(4 \xi^{2}\right), \\
\frac{\omega_{0} \sin \left(\omega_{0} \tau\right)+\beta \cos \left(\omega_{0} \tau\right)}{\omega \cos \left(\omega_{0} \tau\right)-\beta \sin \left(\omega_{0} \tau\right)} \\
=-\frac{\omega_{0}^{2}-\alpha \beta+\alpha \beta \cos \left(\omega_{0} \tau\right)}{\beta \omega_{0}+\alpha \beta \sin \left(\omega_{0} \tau\right)} .
\end{array}\right.
$$

From (45), we have

$$
\begin{aligned}
& \left.A \frac{\mathrm{d} \rho}{\mathrm{d} \mu}\right|_{\mu=\mu^{*}}-\left.B \frac{\mathrm{d} \omega}{\mathrm{d} \mu}\right|_{\mu=\mu^{*}}=C, \\
& \left.B \frac{\mathrm{d} \rho}{\mathrm{d} \mu}\right|_{\mu=\mu^{*}}+\left.A \frac{\mathrm{d} \omega}{\mathrm{d} \mu}\right|_{\mu=\mu^{*}}=D,
\end{aligned}
$$

in which

$$
\begin{aligned}
A= & \beta-\tau \omega_{0} \sin \left(\omega_{0} \tau\right) \sqrt{1+4 \mu^{*} \xi^{2}} \\
& +\cos \left(\omega_{0} \tau\right) \sqrt{1+4 \mu^{*} \xi^{2}}-\tau \cos \left(\omega_{0} \tau\right) \\
& \times\left[\beta \sqrt{1+4 \mu^{*} \xi^{2}}-\alpha \beta\right], \\
B= & 2 \omega_{0}-\tau \omega_{0} \cos \left(\omega_{0} \tau\right) \sqrt{1+4 \mu^{*} \xi^{2}} \\
& -\sin \left(\omega_{0} \tau\right) \sqrt{1+4 \mu^{*} \xi^{2}}+\tau \sin \left(\omega_{0} \tau\right) \\
& \times\left[\beta \sqrt{1+4 \mu^{*} \xi^{2}}-\alpha \beta\right], \\
C= & -\omega_{0} \sin \left(\omega_{0} \tau\right) \frac{2 \xi^{2}}{\sqrt{1+4 \mu^{*} \xi^{2}}} \\
& -\beta \cos \left(\omega_{0} \tau\right) \frac{2 \xi^{2}}{\sqrt{1+4 \mu^{*} \xi^{2}}}, \\
D= & -\omega_{0} \cos \left(\omega_{0} \tau\right) \frac{2 \xi^{2}}{\sqrt{1+4 \mu^{*} \xi^{2}}} \\
& +\beta \sin \left(\omega_{0} \tau\right) \frac{2 \xi^{2}}{\sqrt{1+4 \mu^{*} \xi^{2}}} .
\end{aligned}
$$

Therefore,

$$
\begin{aligned}
\frac{\mathrm{d}}{\mathrm{d} \mu}[\operatorname{Re} \lambda]_{\mu=\mu^{*}} & =\left.\frac{\mathrm{d} \rho}{\mathrm{d} \mu}\right|_{\mu=\mu^{*}}=\frac{\frac{2 \xi^{2}}{\sqrt{1+4 \mu^{*} \xi^{2}}}\left[\beta \omega_{0} \sin \left(\omega_{0} \tau\right)-\left(\beta^{2}+2 \omega_{0}^{2}\right) \cos \left(\omega_{0} \tau\right)-\alpha \tau \beta^{2}\right]+2 \xi^{2}\left(\tau \omega_{0}^{2}-\beta+\tau \beta^{2}\right)}{A^{2}+B^{2}} \\
& \neq 0
\end{aligned}
$$

This implies that the parameter $\mu$ passes through the critical value $\mu^{*}$, there is Hopf bifurcation from the equilibrium $V^{*}$ to a periodic orbit.

Remark 7 It should be noted that in Theorem 11, bifurcation control for small-world network model (2) can be achieved under the TDAS control force (40) with an additional low-pass filter. Unlike the controllers discussed in Sects. 3-5, this controller increases the dimension of the original system. How- ever, it has advantage of stabilizing unstable periodic orbits.

Figure 23 shows the dependence of $\mu^{*}$ upon the feedback gain $\alpha$ according to the controlled model (42) for $\xi=3$ and $\tau=1$. The solid, dotted, dash-dotted curves correspond to a cutoff frequency of $\beta=20,5$, and 1 , respectively. For a small cutoff frequency, i.e., $\beta=1, \mu^{*}$ varies around 0.05 as $\alpha$ increases, which is similar to the case without low-pass 
Fig. 23 The fluctuation of $\mu^{*}$ depending on $\alpha$ for $\xi=3$ and $\tau=1$ as given by controlled system (42)
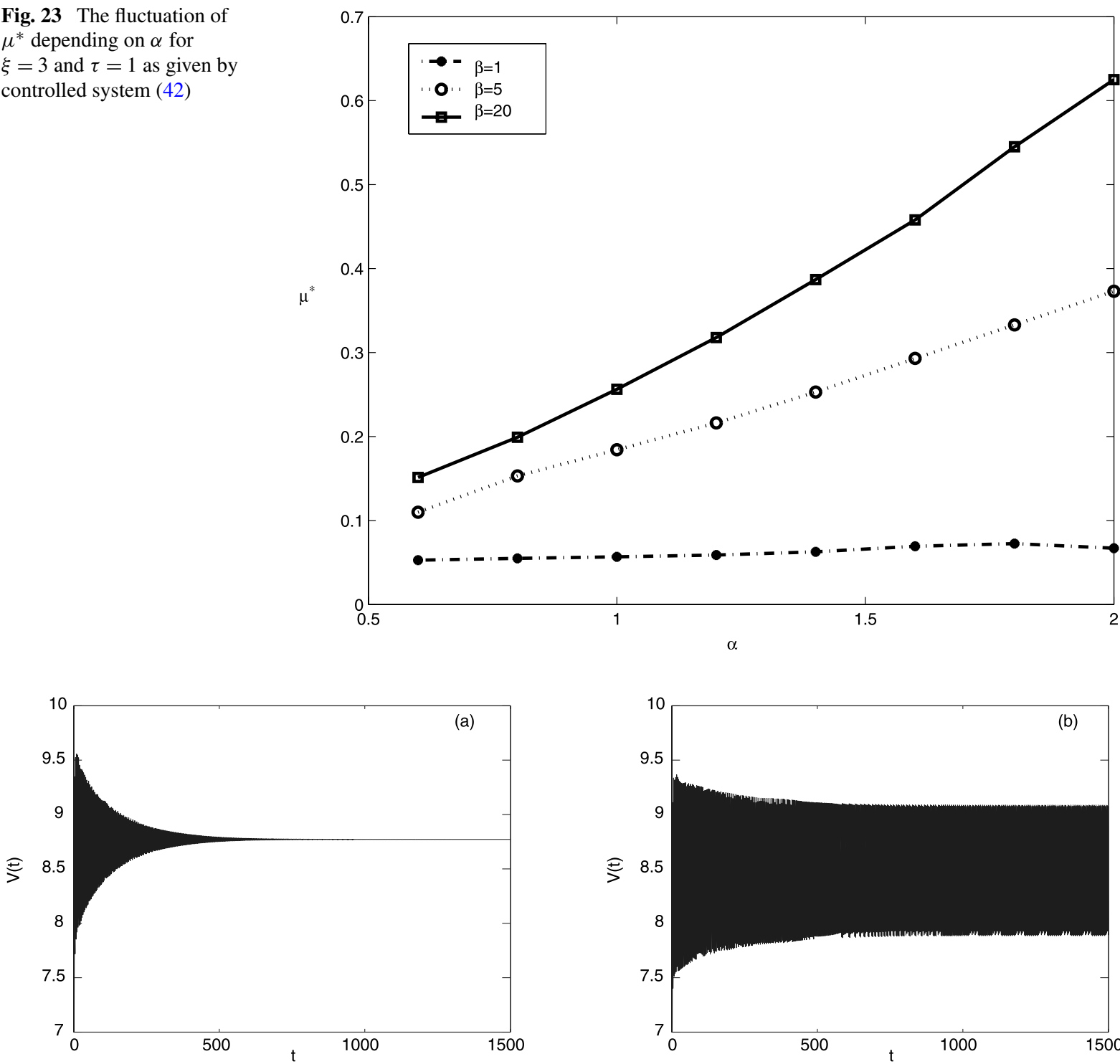

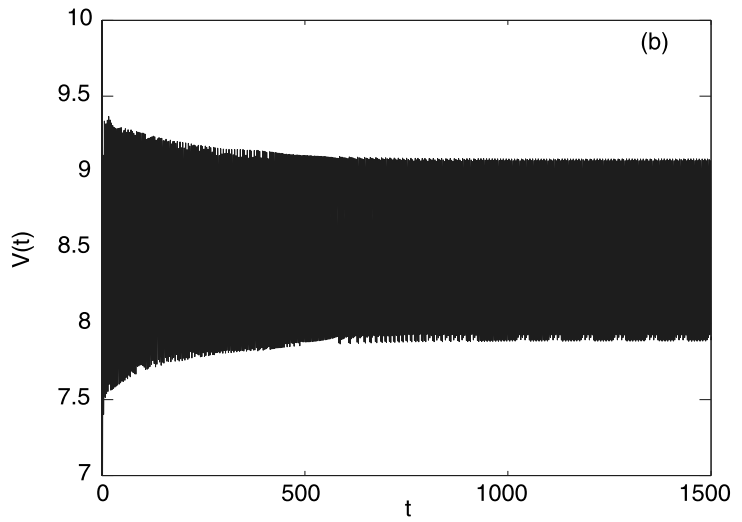

Fig. 24 Waveform plot of controlled model (42) with $\beta=1$ and $\alpha=0.6$ (panel (a): $\mu=0.051 ;$ panel $(\mathbf{b}): \mu=0.053)$

filter (see Figs. 4-6) indicating that the filter has only little effect. For larger $\beta$, however, filtering control signal strengthens the stability and raises the value of $\mu^{*}$. Increasing $\alpha$ postpones the onset of Hopf bifurcation and reduces the instability.

In the case of a combination of $\xi=3, \tau=1$ and $\alpha=0.6$, the values $0.0527,0.1099$ and 0.1512 of $\mu^{*}$ correspond to $\beta=1,5$ and 20 , respectively. Panel (a) of Fig. $24(\beta=1)$ shows trajectories converge to the equilibrium $V^{*}$ at $\mu=0.051$ for the controlled model (42), while panel (b) of Fig. 24 depicts the equi- librium $V^{*}$ is unstable at $\mu=0.053$. A Hopf bifurcation emerges when $\mu$ passes through the $\mu^{*}=0.0527$. Panel (a) of Fig. 25 ( $\beta=5$ ) displays that the equilibrium point $V^{*}$ becomes stable at the value 0.053 of $\mu$ used in panel (b) of Fig. 24. If $\mu=0.13>\mu^{*}=$ 0.1099 , the equilibrium $V^{*}$ loses stability again (see panel (b) of Fig. 25). Further, if one chooses larger value of $\beta$, the controlled small-world network model may have a Hopf bifurcation even for large values of $\mu$ (see Fig. 26). 

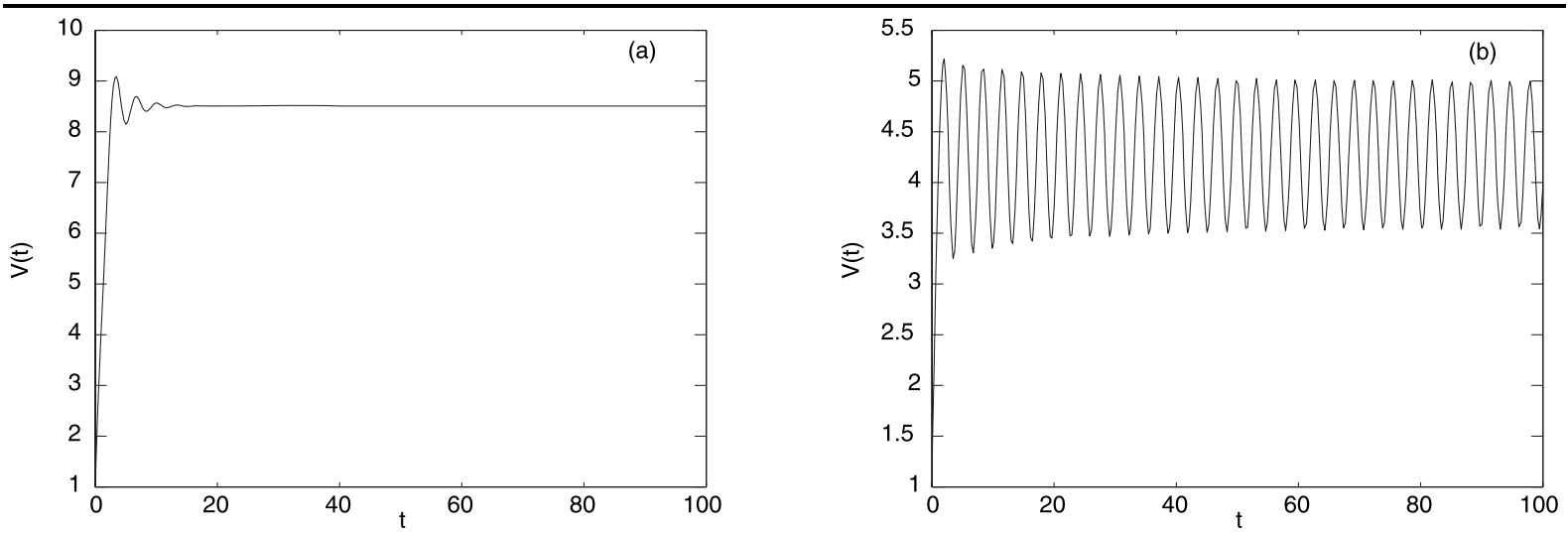

Fig. 25 Waveform plot of controlled model (42) with $\beta=5$ and $\alpha=0.6$ (panel (a): $\mu=0.053$; panel $(\mathbf{b}): \mu=0.13$ )
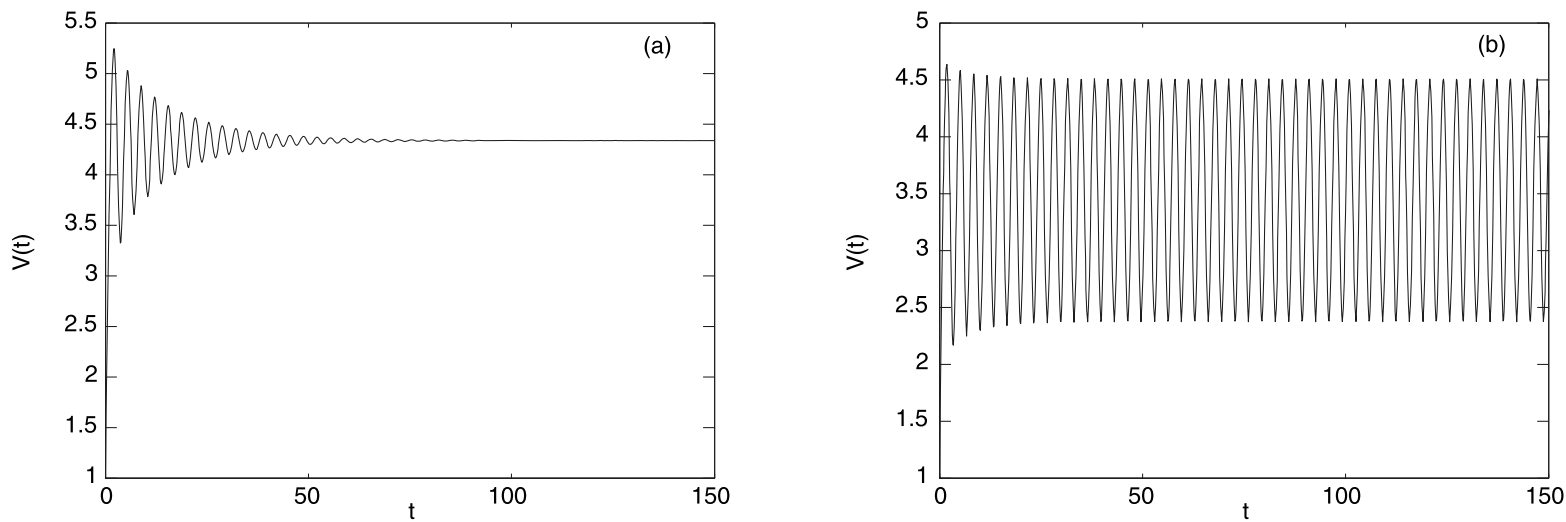

Fig. 26 Waveform plot of controlled model (42) with $\beta=20$ and $\alpha=0.6$ (panel (a): $\mu=0.13$; panel (b): $\mu=0.17$ )

\section{Conclusion}

We have discussed the effects of time-delayed feedback control upon the Hopf bifurcation for a smallworld network model. We have computed the domain of stabilization and the onset of Hopf bifurcation at the equilibrium point. Through the complex analysis, we have derived analytically the main features of the stability domain and the critical value of $\mu^{*}$ by investigating the characteristic equation of the equilibrium point. We find that increasing feedback gain $\alpha$ postpones the onset of Hopf bifurcation and strengthens the stability, and thus a stationary total influenced volume is guaranteed for a large measure parameter $\mu$. For extended time-delayed feedback, we have shown that increasing delay $\tau_{1}$, which is different from original delay $\tau$, increases the critical value of $\mu^{*}$ and reduces the instability. Taking nonzero control loop latencies into account, we have displayed that decrease of latency time raises the value of $\mu^{*}$. Similarly, an additional low-pass filter in the control loop causes a shift of $\mu^{*}$, as well. This suggests that filtering with a cutoff frequency $\beta$ has a similar effect as a latency delay time $\beta^{-1}$. A low-pass filter with large cutoff frequency shifts the onset of Hopf bifurcation to large values. Nevertheless, the described theoretical approach is valid for any dynamical system close to the bifurcation point and allows a complete analytical treatment. We believe that theses results are of general importance for optimizing the control technique of Hopf bifurcation and will stimulate the search for further modifications aiming at the improvement of the control performance.

Up to now, controlling bifurcation via delayed feedback for small-world network model has not yet been fully investigated, which is still open. Due to the finite speed of information processing, various types of time delays, including constant or time-varying de- 
lays, discrete and/or distributed delays, should be considered in modeling small-world network. It has also been realized that the communication transmission in real network systems can be viewed as a noisy process brought on by random fluctuations from probabilistic causes. Thus, stochastic disturbances should be also added into small-world network model. Consequently, the stability analysis problem for various neural networks with stochastic disturbances and time delays has stirred increasing research interests, and relevant results have begun to be published, see e.g. [37-39]. The problem of bifurcation control based on delayed feedback will be studied for small-world network model including stochastic disturbances and/or both discrete and distributed (also called "mixed") time delays in our future works.

Open Access This article is distributed under the terms of the Creative Commons Attribution Noncommercial License which permits any noncommercial use, distribution, and reproduction in any medium, provided the original author(s) and source are credited.

\section{References}

1. Abed, E.H., Wang, H.O., Tesi, A.: Control of bifurcation and chaos. In: Levinen, W.S. (ed.) The Control Handbook, pp. 951-966. CRC Press, Boca Raton (1995)

2. Chen, G.R., Moiola, J.L., Wang, H.O.: Bifurcation control: Theories, methods and applications. Int. J. Bifurc. Chaos 10, 511-548 (2000)

3. Abed, E.H., Fu, J.H.: Local feedback stabilization and bifurcation control: I. Hopf bifurcation. Syst. Control Lett. 7, 11-17 (1986)

4. Chen, G.R., Lu, J., Yap, K.C.: Feedback control of Hopf bifurcations. In: Proc. of IEEE Int. Symp. on Circ. Sys., Monterey, CA (1998)

5. Yu, P., Chen, G.R.: Hopf bifurcation control using nonlinear feedback with polynomial functions. Int. J. Bifurc. Chaos 14, 1683-1704 (2004)

6. Yu, P.: Bifurcation dynamics in control systems. In: Chen, G.R., Hill, D.J., Yu, X. (eds.) Bifurcation Control: Theory and Applications, pp. 99-126. Springer, Berlin (2003)

7. Sieber, J., Krauskopf, B.: Bifurcation analysis of an inverted pendulum with delayed feedback control near a triple-zero eigenvalue singularity. Nonlinearity 17, 85-103 (2004)

8. Bleich, M.E., Socolar, J.E.S.: Stability of periodic orbits controlled by time-delay feedback. Phys. Lett. A 210, 87 94 (1996)

9. Brandt, M.E., Shih, H.T., Chen, G.R.: Linear time-delay feedback control of a pathological rhythm in a cardiac conduction model. Phys. Rev. E 56, 1334-1337 (1997)

10. Song, Y.X., Yu, X.H., Chen, G.R., Xu, J.X., Tian, Y.P.: Time delayed repetitive learning control for chaotic systems. Int. J. Bifurc. Chaos 12, 1057-1065 (2002)
11. Watts, D.J., Strogatz, S.H.: Collective dynamics of smallworld networks. Nature 393, 440-442 (1998)

12. Newman, M.E.J., Watts, D.J.: Scaling and percolation in the small-world network model. Phys. Rev. E 60, 73327342 (1999)

13. Moukarzel, C.F.: Spreading and shortest paths in systems with sparse long-range connections. Phys. Rev. E 60, 62636266 (1999)

14. Newman, M.E.J., Moore, C., Watts, D.J.: Mean-field solution of the small-world network model. Phys. Rev. Lett. 84, 3201-3204 (2000)

15. Wang, X.F., Chen, G.R.: Synchronization in small-world dynamical networks. Int. J. Bifurc. Chaos 12, 187-192 (2002)

16. Wang, X.F., Chen, G.R.: Complex networks: small-world, scale-free, and beyond, IEEE Circuits Syst. Mag. (2003)

17. Yang, X.S.: Chaos in small-world networks. Phys. Rev. E 63, $046206(2001)$

18. Li, C.G., Chen, G.R.: Local stability and Hopf bifurcation in small-world delayed networks. Chaos, Solitons Fractals 20, 353-361 (2004)

19. Chen, G.R. (Ed.): Controlling Chaos and Bifurcations in Engineering Systems. CRC Press, Boca Raton (1999)

20. Pyragas, K.: Continuous control of chaos by selfcontrolling feedback. Phys. Lett. A 170, 421-428 (1992)

21. Cheng, Z.S., Cao, J.: Hopf bifurcation control for delayed complex networks. J. Franklin Inst. 344(6), 846-857 (2007)

22. Hale, J.: Theory of Functional Differential Equations. Springer, New York (1977)

23. Hassard, B.D., Kazarinoff, N.D., Wan, Y.-H.: Theory and Applications of Hopf Bifurcation. Cambridge University Press, Cambridge (1981)

24. Wei, J.J., Ruan, S.G.: Stability and bifurcation in a neural network model with two delays. Phys. D 130, 255-272 (1999)

25. Blakely, J., Illing, L., Gauthier, D.: Controlling fast chaos in delay dynamical systems. Phys. Rev. Lett. 92, 193901 (2004)

26. Sukow, D.W., Bleich, M.E., Gauthier, D.J., Socolar, J.E.S.: Controlling chaos in a fast diode resonator using extended time-delay autosynchronization: Experimental observations and theoretical analysis. Chaos 7, 560-576 (1997)

27. Just, W., Reckwerth, D., Reibold, E., Benner, H.: Influence of control loop latency on time-delayed feedback control. Phys. Rev. E 59, 2826-2829 (1999)

28. Hövel, P., Socolar, J.E.S.: Stability domains for time-delay feedback control with latency. Phys. Rev. E 68, 036206 (2003)

29. Schlesner, J., Amann, A., Janson, N.B., Just, W., Schöll, E.: Self-stabilization of high-frequency oscillations in semiconductor superlattices by time-delayed autosynchronization. Phys. Rev. E 68, 066208 (2003)

30. Pyragas, K., Pyragas, V., Kiss, I.Z., Hudson, J.L.: Adaptive control of unknown unstable steady states of dynamical systems. Phys. Rev. E 70, 026215 (2004)

31. Yap, K.C., Chen, G.R., Ueta, T.: Controlling bifurcations of discrete maps. Lat. Am. Appl. Res. 31, 157-162 (2001)

32. Abed, E.H., Fu, J.H.: Local feedback stabilization and bifurcation control: II. Stationary bifurcation. Syst. Control Lett. 8, 467-473 (1987) 
33. Chen, G.R., Lu, J., Nicholas, B., Ranganathan, S.: Bifurcation dynamics in discrete-time delayed-feedback control systems. Int. J. Bifurc. Chaos 9, 287-293 (1999)

34. Abed, E.H., Wang, H.O., Chen, G.R.: Stabilization of period doubling bifurcations and implications for control of chaos. Phys. D 70, 154-164 (1994)

35. Yaghoobi, H., Abed, E.H.: Local feedback control of the Neimark-Sacker bifurcation. Int. J. Bifurc. Chaos 13, 879893 (2003)

36. Wen, G., Xu, D., Xie, J.: Control of degenerate Hopf bifurcations in three-dimensional maps. Chaos 13, 486-494 (2003)
37. Wang, Z.D., Lauria, S., Fang, J.A., Liu, X.H.: Exponential stability of uncertain stochastic neural networks with mixed time delays. Chaos, Solitons Fractals 32(1), 62-72 (2007)

38. Wang, Z.D., Shu, H.S., Fang, J.A., Liu, X.H.: Robust stability for stochastic Hopfield neural networks with time delays. Nonlinear Anal. Real World Appl. 7(5), 1119-1128 (2006)

39. Wang, Z.D., Liu, Y.R., Liu, X.H.: On global asymptotic stability of neural networks with discrete and distributed delays. Phys. Lett. A 345(4-6), 299-308 (2005) 